\title{
Modular flavor symmetries of three-generation modes on magnetized toroidal orbifolds
}

\author{
Shota Kikuchi, Tatsuo Kobayashi, and Hikaru Uchida \\ Department of Physics, Hokkaido University, Sapporo 060-0810, Japan
}

(Received 15 March 2021; accepted 9 August 2021; published 8 September 2021)

\begin{abstract}
We study the modular symmetry on magnetized toroidal orbifolds with Scherk-Schwarz phases. In particular, we investigate finite modular flavor groups for three-generation modes on magnetized orbifolds. The three-generation modes can be the three-dimensional irreducible representations of covering groups and central extended groups of $\Gamma_{N}$ for $N=3,4,5,7,8,16$, that is, covering groups of $\Delta\left(6(N / 2)^{2}\right)$ for $N=$ even and central extensions of $\operatorname{PSL}\left(2, \mathbb{Z}_{N}\right)$ for $N=$ odd with Scherk-Schwarz phases. We also study anomaly behaviors.
\end{abstract}

DOI: $10.1103 /$ PhysRevD.104.065008

\section{INTRODUCTION}

The origin of the flavor structure, such as quark and lepton masses and their mixing angles, is one of the most significant mysteries in particle physics. Non-Abelian discrete flavor symmetries [1-6] such as $S_{N}, A_{N}, \Delta\left(3 N^{2}\right)$, and $\Delta\left(6 M^{2}\right)$ for the three generations of quarks and leptons are attractive candidates to realize the flavor structure. However, in order to obtain realistic masses and mixing angles of the quarks and leptons, the complicated vacuum alignment of gaugesinglet scalars - the so-called flavons-is required.

The geometries of compact spaces predicted in higherdimensional theories such as superstring theory can be candidates for the origin of the flavor structure (see Refs. $[7,8]$ ). For example, a torus and its orbifold have the complex structure modulus $\tau$, which decides the shape of the torus and the orbifold. There is the modular symmetry $\Gamma \equiv S L(2, \mathbb{Z})$ as well as $\bar{\Gamma} \equiv S L(2, \mathbb{Z}) / \mathbb{Z}_{2}$ as the geometrical symmetry on a torus and some orbifolds. Under the modular transformation, chiral zero modes on the torus and orbifolds, corresponding to the flavors of quarks and leptons, are transformed; that is, the modular symmetry can be regarded as the flavor symmetry. In addition, Yukawa couplings as well as higher-order couplings can be functions of the modulus $\tau$, and then they also transform under the modular transformation since they can be obtained by overlap integrals of the zero-mode profiles on the torus and orbifolds. Instead of flavons, a vacuum expectation value of the modulus $\tau$ breaks the flavor symmetry and characterizes the flavor structure. These features are different from ones in the conventional

Published by the American Physical Society under the terms of the Creative Commons Attribution 4.0 International license. Further distribution of this work must maintain attribution to the author(s) and the published article's title, journal citation, and DOI. Funded by SCOAP ${ }^{3}$. flavor models. The modular transformation behavior of zero modes was investigated in magnetized D-brane models [9-15] and heterotic orbifold models [16-20] (see also Refs. [21-23]). In particular, on magnetized $T^{2}$ with the magnetic flux $M$, there are $M$ chiral zero modes [24], and in a recent work [13] it was shown that the zero modes with $M=$ even and vanishing Scherk-Schwarz (SS) phases behave as modular forms of weight $1 / 2$, and then they transform as $M$-dimensional representations of the finite modular subgroup $\tilde{\Gamma}_{2 M}$, which is the quadruple covering group of $\Gamma_{2 M}$. There also exists the modular symmetry on the magnetized $T^{2} / \mathbb{Z}_{2}^{(\mathrm{t})}$ twisted orbifold. The number of zero modes on the magnetized $T^{2} / \mathbb{Z}_{2}^{(\mathrm{t})}$ twisted orbifold was investigated in Refs. [25-28]. Similarly, in Ref. [14] it was shown that zero modes on the magnetized $T_{1}^{2} \times T_{2}^{2}$ with the magnetic fluxes $M^{(i)}(i=1,2)$ on $T_{i}^{2}$ and its orbifolds ${ }^{1}$ behave as modular forms of weight 1 and they transform under the finite modular subgroup $\Gamma_{2 \mathrm{lcm}\left(M^{(1)}, M^{(2)}\right)}^{\prime}$, which is the double covering group of $\Gamma_{21 \mathrm{~cm}\left(M^{(1)}, M^{(2)}\right)}$. The number of zero modes was investigated in Ref. [15]. The modular transformation for Yukawa couplings was also studied in Ref. [15]. Thus, it is important to study the modular flavor symmetries, particularly in magnetized orbifold models.

Furthermore, the finite modular subgroups $\Gamma_{N}$ for $N=2$, $3,4,5$ are isomorphic to $S_{3}, A_{4}, S_{4}, A_{5}$, respectively [29]. Similarly, $\Gamma_{N}^{\prime}$ for $N=3,4,5$ are isomorphic to $T^{\prime}, S_{4}^{\prime}, A_{5}^{\prime}$, respectively [30]. These results are well motivated for realistic model building. In particular, in Ref. [29] threedimensional irreducible representations were studied in

\footnotetext{
${ }^{1}$ Both of moduli on $T_{i}^{2}, \tau_{i}$, are identified each other, i.e., $\tau_{1}=\tau_{2} \equiv \tau$. Such moduli identification can be realized by certain three-form fluxes [23] or $\mathbb{Z}_{2}^{(\mathrm{p})}$ permutations.
} 
the finite modular subgroups and it was shown that three-dimensional irreducible representations appear only in the finite modular subgroups: $\Gamma_{3} \simeq \operatorname{PSL}\left(2, \mathbb{Z}_{3}\right) \simeq A_{4}$, $\Gamma_{4} \simeq S_{4}, \quad \Gamma_{5} \simeq \operatorname{PSL}\left(2, \mathbb{Z}_{5}\right) \simeq A_{5}, \quad \Gamma_{7} \simeq \operatorname{PSL}\left(2, \mathbb{Z}_{7}\right)$, $\Gamma_{8} \supset \Delta(96)$, and $\Gamma_{16} \supset \Delta(384)$. Note that a triplet representation of $\Gamma_{8}\left[\Gamma_{16}\right]$ is not faithful, but rather represents its subgroup $\Delta(96)$ [ $\Delta(384)$ ] [29]. Recently, the bottom-up approach of model building with the modular flavor symmetries was studied extensively for $\Gamma_{N}$ [31] and for its covering groups $[30,32]$.

In this paper, we study modular flavor groups of the three-generation modes on magnetized orbifolds. We study nonvanishing SS phases, although previous studies on the modular symmetry did not include SS phases. We find that the three-generation modes are the three-dimensional representations of corresponding covering groups and central extended groups of the above finite modular subgroups provided in Ref. [29].

After this paper was completed, relevant papers appeared [33,34]. In Ref. [33] it was claimed that the violation of the modular symmetry in models with odd magnetic fluxes is strange and it is inconsistent. To preserve the modular symmetry, a certain shift of the coordinate was introduced in the models with odd magnetic fluxes in Ref. [33], that is, one class of compactification. However, the modular symmetry can break when we impose further boundary conditions on wave functions by geometry and/or gauge background, that is, a generic compactification. For example, $T^{2} / \mathbb{Z}_{N}$ orbifolds with $N=3,4,6$ break the modular symmetry, while some residual symmetries remain. The full modular symmetry remains in wave functions on $T^{2}$ and $T^{2} / \mathbb{Z}_{2}$ with even magnetic fluxes and vanishing Wilson lines (WLs), which are equivalent to SS phases. However, nonvanishing SS phases can break the modular symmetry for even magnetic fluxes. Indeed, the number of zero modes depends on the SS phases $[26,28]$. On the other hand, the modular symmetry is broken in wave functions for odd magnetic fluxes and vanishing Wilson lines and SS phases, but the modular symmetry remains for odd magnetic fluxes and nonvanishing WLs, which is a discrete shift of the coordinate. This result is consistent with Ref. [33]. At any rate, a general class of compactifications can be decomposed into two classes. One class of compactifications preserves the modular symmetry, while the other class breaks the modular symmetry. Both are consistent compactifications. Thus, one can concentrate on the compactification preserving the modular symmetry, or one can discuss generic compactification including breaking of the modular symmetry. In Ref. [34] SS phases were also studied.

This paper is organized as follows. In Sec. II we review the modular symmetry on magnetized $T^{2}$ and $T^{2} / \mathbb{Z}_{2}^{(\mathrm{t})}$ twisted orbifolds without the SS phases. In Sec. III we study the modular symmetry on magnetized $T^{2}$ and $T^{2} / \mathbb{Z}_{2}^{(\mathrm{t})}$ twisted orbifolds with the SS phases. We can consider the modular symmetry of not only wave functions with magnetic flux $M=$ even and vanishing SS phases, but also ones with magnetic flux $M=$ odd and certain SS phases. In Sec. IV we show the specific modular flavor groups for three-generation modes on magnetized $T^{2} / \mathbb{Z}_{2}^{(\mathrm{t})}$ twisted orbifolds with the SS phases. We find that the three-generation modes are the three-dimensional representations of the quadruple covering groups and $Z_{8}$ central extended groups of the corresponding modular flavor groups provided in Ref. [29]. We also extend the analyses to the modular symmetry on magnetized $T_{1}^{2} / \mathbb{Z}_{2}^{\left(\mathrm{t}_{1}\right)} \times$ $T^{2} / \mathbb{Z}_{2}^{\left(\mathrm{t}_{2}\right)}$ orbifolds and the $\mathbb{Z}_{2}^{(\mathrm{p})}$ permutation orbifold, i.e., $\left(T_{1}^{2} \times T_{2}^{2}\right) /\left(\mathbb{Z}_{2}^{(\mathrm{t})} \times \mathbb{Z}_{2}^{(\mathrm{p})}\right)$ orbifolds in Secs. V and VI. We obtain three-dimensional representations of all of the double covering groups of $\Gamma_{N}$ for $N=4,8,16$, i.e., covering groups of $\Delta\left(6 N^{\prime 2}\right)$ with $N^{\prime}=N / 2$, and $Z_{4}$ central extended groups of $\Gamma_{N}$ for $N=3,5$, 7, i.e., $Z_{4}$ extensions of $\operatorname{PSL}\left(2, \mathbb{Z}_{N}\right)$. In Sec. VII we conclude this study. In Appendix A we review that the SS phases can be replaced by the WLs through a gauge transformation and we show that the modular transformations for them are consistent. In Appendix B we also show that the $\mathbb{Z}_{N}$ SS phases are related to the $\mathbb{Z}_{N}$ shift modes. In Appendix $\mathrm{C}$ we prove that $\tilde{\Delta}\left(6 M^{2}\right)$, which is the quadruple covering group of $\Delta\left(6 M^{2}\right)$, can be obtained. In Appendix D we express the three-dimensional modular forms obtained from the wave functions on magnetized orbifolds.

\section{MODULAR SYMMETRY ON MAGNETIZED $T^{2}$ AND $T^{2} / \mathbb{Z}_{2}^{(\mathbf{t})}$ TWISTED ORBIFOLDS WITHOUT THE SCHERK-SCHWARZ PHASES}

In this section we review the modular symmetry on magnetized $T^{2}$ and $T^{2} / \mathbb{Z}_{2}^{(\mathrm{t})}$ twisted orbifolds without the SS phases.

First, we review the modular symmetry of $T^{2}$ [35-38]. A two-dimensional torus $T^{2}$ can be constructed as $T^{2} \simeq \mathbb{C} / \Lambda$, where $\Lambda$ is a two-dimensional lattice spanned by lattice vectors $e_{k}(k=1,2)$. The torus is characterized by the complex structure modulus $\tau \equiv e_{2} / e_{1}(\operatorname{Im} \tau>0)$. We also define the complex coordinate of $\mathbb{C}$ as $u$ and that of $T^{2}$ as $z \equiv u / e_{1}$, so that $z+1$ and $z+\tau$ are identified with $z$. The metric on $T^{2}$ is given by

$$
d s^{2}=2 h_{\mu \nu} d z^{\mu} d \bar{z}^{\nu}, \quad h=\left|e_{1}\right|^{2}\left(\begin{array}{cc}
0 & \frac{1}{2} \\
\frac{1}{2} & 0
\end{array}\right),
$$

and then the area of $T^{2}$ is $\mathcal{A}=\left|e_{1}\right|^{2} \operatorname{Im} \tau$.

Here, we can consider the same lattice spanned by the following lattice vectors transformed by $S L(2, \mathbb{Z}) \equiv \Gamma$ :

$$
\left(\begin{array}{l}
e_{2}^{\prime} \\
e_{1}^{\prime}
\end{array}\right)=\left(\begin{array}{ll}
a & b \\
c & d
\end{array}\right)\left(\begin{array}{l}
e_{2} \\
e_{1}
\end{array}\right), \quad \gamma=\left(\begin{array}{ll}
a & b \\
c & d
\end{array}\right) \in S L(2, \mathbb{Z}) \equiv \Gamma .
$$


The $S L(2, \mathbb{Z})$ is generated by

$$
S=\left(\begin{array}{cc}
0 & 1 \\
-1 & 0
\end{array}\right), \quad T=\left(\begin{array}{ll}
1 & 1 \\
0 & 1
\end{array}\right),
$$

and they satisfy the following algebraic relations:

$$
Z \equiv S^{2}=-\mathbb{I}, \quad Z^{2}=S^{4}=(S T)^{3}=\mathbb{I} .
$$

Under the $S L(2, \mathbb{Z})$ transformation, the complex coordinate of the torus $z$ and the complex structure modulus $\tau$ are transformed as

$$
\gamma:(z, \tau) \rightarrow(\gamma(z, \tau))=\left(\frac{z}{c \tau+d}, \frac{a \tau+b}{c \tau+d}\right) .
$$

The above transformation for the modulus $\tau$ is called the (inhomogeneous) modular transformation, and $\bar{\Gamma} \equiv$ $\Gamma /\{ \pm \mathbb{I}\}$ is called the (inhomogeneous) modular group since $\tau$ is invariant under $Z=-\mathbb{I}$.

We define the principal congruence subgroup $\Gamma(N)$ of level $N$ by

$$
\begin{aligned}
\Gamma(N) & \equiv\left\{h=\left(\begin{array}{ll}
a^{\prime} & b^{\prime} \\
c^{\prime} & d^{\prime}
\end{array}\right)\right. \\
& \left.\in \Gamma \mid\left(\begin{array}{ll}
a^{\prime} & b^{\prime} \\
c^{\prime} & d^{\prime}
\end{array}\right) \equiv\left(\begin{array}{ll}
1 & 0 \\
0 & 1
\end{array}\right)(\bmod N)\right\} .
\end{aligned}
$$

Then, the modular forms $f(\tau)$ of the (integral) weight $k$ for $\Gamma(N)$ are the holomorphic functions of $\tau$, which transform under the modular transformation in Eq. (5) as

$$
\begin{aligned}
f(\gamma(\tau)) & =J_{k}(\gamma, \tau) \rho(\gamma) f(\tau), \quad J_{k}(\gamma, \tau)=(c \tau+d)^{k}, \\
\gamma(\tau) & =\frac{a \tau+b}{c \tau+d}, \quad \gamma=\left(\begin{array}{ll}
a & b \\
c & d
\end{array}\right) \in \Gamma .
\end{aligned}
$$

Here, $\rho(\gamma)$ denotes the unitary representation of the quotient group $\Gamma_{N}^{\prime} \equiv \Gamma / \Gamma(N)$ satisfying the following algebraic relations:

$$
\begin{gathered}
\rho(Z)=\rho(S)^{2}=(-1)^{k} \mathbb{I}, \\
\rho(Z)^{2}=\rho(S)^{4}=[\rho(S) \rho(T)]^{3}=\mathbb{I}, \\
\rho(Z) \rho(T)=\rho(T) \rho(Z), \\
\rho(T)^{N}=\mathbb{I} .
\end{gathered}
$$

For even weight $k$, in particular, $\rho(\gamma)$ becomes the unitary representation of the quotient group $\Gamma_{N} \equiv \bar{\Gamma} / \bar{\Gamma}(N)$, where $\bar{\Gamma}(N) \equiv \Gamma(N) /\{ \pm \mathbb{I}\}$ for $N=1,2,{ }^{2}$ and $\bar{\Gamma}(N) \equiv \Gamma(N)$ for

\footnotetext{
${ }^{2}$ Since $Z=-\mathbb{I} \in \Gamma(N)$ for $N=1,2, \rho(Z)=\mathbb{I}$ should be satisfied and then the modular weight $k$ should be even.
}

$N>2$. Note that $\Gamma_{N}$ for $N=2,3,4$, and 5 are isomorphic to $S_{3}, A_{4}, S_{4}$, and $A_{5}$, respectively [29], and $\Gamma_{N}^{\prime}$ for $N=3$, 4 , and 5 are isomorphic to the corresponding double covering groups $T^{\prime}, S_{4}^{\prime}$, and $A_{5}^{\prime}$, respectively [30]. In what follows, we review the wave functions of $(z, \tau)$ on a magnetized torus and then review their behavior as modular forms under the modular transformation in Eq. (5).

First, let us review the wave functions, particularly the zero-mode wave functions of the two-dimensional spinor, on the torus with $U(1)$ magnetic flux [24]. Here, we do not consider the WLs or the SS phases. In the next section, we will study the case with nonvanishing SS phases. ${ }^{3}$ The $U(1)$ magnetic flux is given by

$$
F=\frac{\pi i M}{\operatorname{Im} \tau} d z \wedge d \bar{z}
$$

which satisfies the quantization condition $(2 \pi)^{-1} \int_{T^{2}} F=$ $M \in \mathbb{Z}$. This flux is induced by the vector potential

$$
A(z)=\frac{\pi M}{\operatorname{Im} \tau} \operatorname{Im}(\bar{z} d z) .
$$

This vector potential transforms under lattice translations as

$$
\begin{aligned}
& A(z+1)=A(z)+d\left(\frac{\pi M}{\operatorname{Im} \tau} \operatorname{Im} z\right)=A(z)+d \chi_{1}(z), \\
& A(z+\tau)=A(z)+d\left(\frac{\pi M}{\operatorname{Im} \tau} \operatorname{Im} \bar{\tau} z\right)=A(z)+d \chi_{2}(z),
\end{aligned}
$$

which correspond to a $U(1)$ gauge transformation. Thereby, the two-dimensional spinor with $U(1)$ unit charge $q=1$,

$$
\psi(z, \tau)=\left(\begin{array}{l}
\psi_{+}(z, \tau) \\
\psi_{-}(z, \tau)
\end{array}\right),
$$

should satisfy the following boundary conditions:

$$
\begin{aligned}
& \psi(z+1, \tau)=e^{i \chi_{1}(z)} \psi(z, \tau)=e^{\pi i M \frac{\operatorname{Im} z}{\operatorname{m} \tau} \tau(z, \tau),} \\
& \psi(z+\tau, \tau)=e^{i \chi_{2}(z)} \psi(z, \tau)=e^{\pi i M \frac{\operatorname{Im} \tilde{z} z}{\operatorname{Im} \tau}} \psi(z, \tau) .
\end{aligned}
$$

Under these boundary conditions, we can solve the zeromode Dirac equation,

$$
i \not D \psi(z, \tau)=0
$$

and then only $\psi_{+}(z, \tau)\left(\psi_{-}(z, \tau)\right)$ has $|M|$ degenerate zero modes when $M$ is positive (negative). In what follows, we

\footnotetext{
${ }^{3}$ The WLs can be replaced by the SS phases [26]. We review this and also show its consistency in terms of the modular symmetry in Appendix A.
} 
consider the positive flux $M$. The $j$ th zero-mode wave function on the torus with the flux $M$ is expressed as

$$
\begin{aligned}
\psi_{T^{2}}^{j, M}(z, \tau)= & \left(\frac{M}{\mathcal{A}^{2}}\right)^{1 / 4} e^{\pi i M z \frac{\mathrm{m} z}{\operatorname{mm}} \vartheta}\left[\begin{array}{c}
\frac{j}{M} \\
0
\end{array}\right](M z, M \tau), \\
& \forall j \in \mathbb{Z}_{M}=\{0,1,2, \ldots, M-1\},
\end{aligned}
$$

where $\vartheta$ denotes the Jacobi theta function defined as

$$
\vartheta\left[\begin{array}{l}
a \\
b
\end{array}\right](\nu, \tau)=\sum_{l \in \mathbb{Z}} e^{\pi i(a+l)^{2} \tau} e^{2 \pi i(a+l)(\nu+b)} .
$$

We take the following normalization condition:

$$
\int_{T^{2}} d z d \bar{z}\left(\psi_{T^{2}}^{j, M}(z, \tau)\right)^{*} \psi_{T^{2}}^{k, M}(z, \tau)=(2 \operatorname{Im} \tau)^{-1 / 2} \delta_{j, k} .
$$

Now, we can see that the wave functions for $\forall j$ in Eq. (18) behave as modular forms of weight $1 / 2^{4}$ under the modular transformation in Eq. (5) [13] as follows. We first introduce the double covering group of $\Gamma$,

$$
\tilde{\Gamma} \equiv\{[\gamma, \epsilon] \mid \gamma \in \Gamma, \epsilon \in\{ \pm 1\}\} .
$$

The generators are given by

$$
\tilde{S} \equiv[S, 1], \quad \tilde{T} \equiv[T, 1],
$$

and they satisfy the following algebraic relations:

$$
\begin{aligned}
\tilde{Z} & \equiv \tilde{S}^{2}, \quad \tilde{Z}^{2}=\tilde{S}^{4}=(\tilde{S} \tilde{T})^{3}=[\mathbb{I},-1], \\
\tilde{Z}^{4} & =\tilde{S}^{8}=(\tilde{S} \tilde{T})^{6}=[\mathbb{I}, 1] \equiv \mathbb{I}, \quad \tilde{Z} \tilde{T}=\tilde{T} \tilde{Z} .
\end{aligned}
$$

Note that the modular transformation in Eq. (5) does not change upon replacing $\gamma \in \Gamma$ with $\tilde{\gamma} \equiv[\gamma, \epsilon] \in \tilde{\Gamma}$. We also introduce the congruence subgroup,

$$
\tilde{\Gamma}(N) \equiv\{[h, \epsilon] \in \tilde{\Gamma} \mid h \in \Gamma(N), \epsilon=1\} .
$$

Then, the modular forms $f(\tau)$ of the (half integral) weight $k / 2$ for $\tilde{\Gamma}(N)$ transform under the modular transformation as

$$
\begin{gathered}
f(\tilde{\gamma}(\tau))=\tilde{J}_{k / 2}(\tilde{\gamma}, \tau) \tilde{\rho}(\tilde{\gamma}) f(\tau), \quad \tilde{\gamma} \in \tilde{\Gamma}, \\
\tilde{J}_{k / 2}(\tilde{\gamma}, \tau)=\epsilon^{k} J_{k / 2}(\gamma, \tau)=\epsilon^{k}(c \tau+d)^{k / 2}, \quad k \in \mathbb{Z},
\end{gathered}
$$

where $\tilde{\rho}(\tilde{\gamma})$ is the unitary representation of the quotient group $\tilde{\Gamma}_{N} \equiv \tilde{\Gamma} / \tilde{\Gamma}(N)$, which is the double covering group of $\Gamma_{N}^{\prime}$, satisfying the following algebraic relations:

\footnotetext{
${ }^{4}$ For details see, e.g., Refs. [36,39,40].
}

$$
\begin{gathered}
\tilde{\rho}(\tilde{Z})=\tilde{\rho}(\tilde{S})^{2}=e^{\pi i k / 2} \mathbb{I}, \\
\tilde{\rho}(\tilde{Z})^{2}=\tilde{\rho}(\tilde{S})^{4}=[\tilde{\rho}(\tilde{S}) \tilde{\rho}(\tilde{T})]^{3}=e^{\pi i k} \mathbb{I}, \\
\tilde{\rho}(\tilde{Z})^{4}=\tilde{\rho}(\tilde{S})^{8}=[\tilde{\rho}(\tilde{S}) \tilde{\rho}(\tilde{T})]^{6}=\mathbb{I} \\
\tilde{\rho}(\tilde{Z}) \tilde{\rho}(\tilde{T})=\tilde{\rho}(\tilde{T}) \tilde{\rho}(\tilde{Z}) \\
\tilde{\rho}(\tilde{T})^{N}=\mathbb{I} .
\end{gathered}
$$

Here we take $(-1)^{k / 2}=e^{-\pi i k / 2}$. On the other hand, the wave functions for $\forall j$ in Eq. (18) transform under the modular transformation as

$\psi_{T^{2}}^{j, M}(\tilde{\gamma}(z, \tau))=\tilde{J}_{1 / 2}(\tilde{\gamma}, \tau) \sum_{k=0}^{M-1} \tilde{\rho}_{T^{2}}(\tilde{\gamma})_{j k} \psi_{T^{2}}^{k, M}(z, \tau), \quad \tilde{\gamma} \in \tilde{\Gamma}$,

$\tilde{\rho}_{T^{2}}(\tilde{S})_{j k}=e^{\pi i / 4} \frac{1}{\sqrt{M}} e^{2 \pi i \frac{i k}{M}}, \quad \tilde{\rho}_{T^{2}}(\tilde{T})_{j k}=e^{\pi i^{i^{2}}} \delta_{j, k}$,

where $\tilde{\rho}_{T^{2}}(\tilde{\gamma})$ satisfies Eqs. (27)-(31) with $k / 2=1 / 2$ and $N=2 M$, although $\mathbb{I}_{j k}=\delta_{j, k}$ in Eq. (27) is modified into $\delta_{M-j, k}$, derived from

$$
\psi_{T^{2}}^{j, M}(\tilde{Z}(z, \tau))=\psi_{T^{2}}^{j, M}(-z, \tau)=\psi_{T^{2}}^{M-j, M}(z, \tau) .
$$

Note that the above modular transformation for the wave functions without the SS phases can be valid only if the magnetic flux $M$ is even because of the consistency of the boundary conditions in Eqs. (15) and (16) under the $T$ transformation. That is, the wave functions after the $T$ transformation satisfy

$$
\psi(z+\tau+1, \tau+1)=e^{\pi i M \frac{\operatorname{lm}(\bar{z}+1) z}{\operatorname{Im} \tau}} \psi(z, \tau+1),
$$

while the wave functions before the $T$ transformation satisfy

$$
\psi(z+\tau+1, \tau)=e^{-\pi i M} e^{\pi i M \frac{\operatorname{Im}(\bar{\tau}+1) z}{\operatorname{Im} \tau}} \psi(z, \tau) .
$$

In the next section, however, we will show that when we take the SS phases into account, we can also consider the modular transformation for wave functions with the flux $M=$ odd. Thus, the wave functions on $T^{2}$ with the magnetic flux $M \in 2 \mathbb{Z}$ and vanishing SS phases behave as the modular forms of weight $1 / 2$ for $\tilde{\Gamma}(2 M)$. They seem to be a $M$-dimensional representation. However, they can be a reducible representation. Their concrete flavor symmetry depends on irreducible representations. For example, they cannot be faithful. Thus, we will study concrete flavor symmetries of zero modes in the following sections. 
Finally, we also review the zero-mode wave functions on the magnetized $T^{2} / \mathbb{Z}_{2}^{(\mathrm{t})}$ twisted orbifold without the SS phases [25] and the modular transformation for them [13] (see also Refs. [9,10]). The $T^{2} / \mathbb{Z}_{2}^{(\mathrm{t})}$ twisted orbifold can be obtained by further identifying the $\mathbb{Z}_{2}^{(\mathrm{t})}$ twisted point $-z$ with $z$. Note that the modulus $\tau$ is not restricted by $\mathbb{Z}_{2}^{(\mathrm{t})}$ twist orbifolding, which means we can also consider the modular transformation on the $T^{2} / \mathbb{Z}_{2}^{(\mathrm{t})}$ twisted orbifold. Then, the wave functions on the magnetized $T^{2} / \mathbb{Z}_{2}^{(\mathrm{t})}$ twisted orbifold should also satisfy the boundary condition

$$
\psi_{T^{2} / \mathbb{Z}_{2}^{(\mathrm{t}) m}}^{j, M}(-z, \tau)=(-1)^{m} \psi_{T^{2} / \mathbb{Z}_{2}^{(\mathrm{t}) m}}^{j, M}(z, \tau), \quad m \in \mathbb{Z}_{2}^{(\mathrm{t})}
$$

in addition to the boundary conditions on the magnetized $T^{2}$ in Eqs. (15) and (16). Actually, their boundary conditions are satisfied by the following linear combination of wave functions on the magnetized $T^{2}$ :

$$
\psi_{T^{2} / \mathbb{Z}_{2}^{(\mathrm{t}) m}}^{j, M}(z, \tau)=\mathcal{N}_{(\mathrm{t})}^{j}\left(\psi_{T^{2}}^{j, M}(z, \tau)+(-1)^{m} \psi_{T^{2}}^{j, M}(-z, \tau)\right),
$$

where $\mathcal{N}_{(\mathrm{t})}^{j}$ denotes the normalization factor determined by the normalization condition in Eq. (20). Since the wave functions on the $T^{2}$ without the SS phases satisfy Eq. (34), those on the $T^{2} / \mathbb{Z}_{2}^{(\mathrm{t})}$ twisted orbifold without the SS phases can be expanded by

$$
\begin{aligned}
\psi_{T^{2} / \mathbb{Z}_{2}^{(\mathrm{t}) m}}^{j, M}(z, \tau) & =\mathcal{N}_{(\mathrm{t})}^{j} \sum_{k=0}^{M-1}\left(\delta_{j, k}+(-1)^{m} \delta_{M-j, k}\right) \psi_{T^{2}}^{k, M}(z, \tau), \\
\mathcal{N}_{(\mathrm{t})}^{j} & = \begin{cases}1 / 2 & (j=0, M / 2), \\
1 / \sqrt{2} & (\text { otherwise }) .\end{cases}
\end{aligned}
$$

Then, the number of zero modes for even $M$ are equal to $M / 2+1$ for $\mathbb{Z}_{2}^{(\mathrm{t})}$-even modes $(m=0)$ and $M / 2-1$ for $\mathbb{Z}_{2}^{(\mathrm{t})}$-odd modes $(m=1)$. Furthermore, under the modular transformation, these transform similarly to Eq. (32) by replacing Eq. (33) with

$$
\begin{aligned}
& \tilde{\rho}_{T^{2} / \mathbb{Z}_{2}^{(\mathrm{t}) 0}}(\tilde{S})_{j k}=\mathcal{N}_{(\mathrm{t})}^{j} \mathcal{N}_{(\mathrm{t})}^{k} \frac{4 e^{\pi i / 4}}{\sqrt{M}} \cos \left(\frac{2 \pi j k}{M}\right), \\
& \tilde{\rho}_{T^{2} / \mathbb{Z}_{2}^{(\mathrm{t}) 0}}(\tilde{T})_{j k}=e^{\pi i i_{M}^{2}} \delta_{j, k}, \\
& \tilde{\rho}_{T^{2} / \mathbb{Z}_{2}^{(\mathrm{t}) 1}}(\tilde{S})_{j k}=\mathcal{N}_{(\mathrm{t})}^{j} \mathcal{N}_{(\mathrm{t})}^{k} \frac{4 i e^{\pi i / 4}}{\sqrt{M}} \sin \left(\frac{2 \pi j k}{M}\right), \\
& \tilde{\rho}_{T^{2} / \mathbb{Z}_{2}^{(\mathrm{t}) 1}}(\tilde{T})_{j k}=e^{\pi i i_{M}^{2}} \delta_{j, k},
\end{aligned}
$$

where $\tilde{\rho}_{T^{2} / \mathbb{Z}_{2}^{(\mathrm{t}) m}}(\tilde{\gamma})$ for each $m \in \mathbb{Z}_{2}$ satisfies Eqs. (27)-(31) with $k / 2=1 / 2$ and $N=2 M$, although $\mathbb{I}_{j k}=\delta_{j, k}$ in Eq. (27) is modified into $(-1)^{m} \delta_{j, k}$, derived from Eq. (37). Thus, both the $\mathbb{Z}_{2}^{(\mathrm{t})}$-even and -odd mode wave functions on the $T^{2} / \mathbb{Z}_{2}^{(\mathrm{t})}$ twisted orbifold with magnetic flux $M \in 2 \mathbb{Z}$ and vanishing SS phases behave as modular forms of weight $1 / 2$. They decompose into $(M / 2+1)$ - and $(M / 2-1)$-dimensional representations for $\mathbb{Z}_{2}^{(\mathrm{t})}$-even and -odd modes, respectively. That is, the representations on the magnetized $T^{2}$ can be decomposed into smaller representations on the magnetized $T^{2} / \mathbb{Z}_{2}^{(\mathrm{t})}$ twisted orbifold. We will study their concrete flavor symmetries in the following sections.

\section{MODULAR SYMMETRY ON MAGNETIZED $T^{2}$ AND $T^{2} / \mathbb{Z}_{2}^{(\mathbf{t})}$ TWISTED ORBIFOLDS WITH THE SCHERK-SCHWARZ PHASES}

In this section we review the wave functions on magnetized $T^{2}$ and $T^{2} / \mathbb{Z}_{2}^{(\mathrm{t})}$ twisted orbifolds with SS phases [26], and then we study their modular symmetry.

The wave functions on $T^{2}$ with flux $M$ and SS phases $\left(\alpha_{1}, \alpha_{2}\right)\left(0 \leq \alpha_{1}, \alpha_{2}<1\right)^{5}$ satisfy the boundary conditions

$$
\begin{aligned}
\psi^{\alpha_{1}, \alpha_{2}}(z+1, \tau) & =e^{2 \pi i \alpha_{1}} e^{i \chi_{1}(z)} \psi^{\alpha_{1}, \alpha_{2}}(z, \tau) \\
& =e^{2 \pi i \alpha_{1}} e^{\pi i M_{\operatorname{Im} z} \tau} \psi^{\alpha_{1}, \alpha_{2}}(z, \tau), \\
\psi^{\alpha_{1}, \alpha_{2}}(z+\tau, \tau) & =e^{2 \pi i \alpha_{2}} e^{i \chi_{2}(z)} \psi^{\alpha_{1}, \alpha_{2}}(z, \tau) \\
& =e^{2 \pi i \alpha_{2}} e^{\pi i M \frac{\operatorname{Im} \bar{z} z}{\operatorname{lm} \tau}} \psi^{\alpha_{1}, \alpha_{2}}(z, \tau)
\end{aligned}
$$

instead of Eqs. (15) and(16). Then, the $j$ th zero-mode wave function is expressed as

$$
\begin{aligned}
\psi_{T^{2}}^{\left(j+\alpha_{1}, \alpha_{2}\right), M}(z, \tau)= & \left(\frac{M}{\mathcal{A}^{2}}\right)^{1 / 4} e^{\pi i M z \frac{\operatorname{lm} z}{\ln z} \vartheta}\left[\begin{array}{c}
\frac{j+\alpha_{1}}{M} \\
-\alpha_{2}
\end{array}\right](M z, M \tau), \\
& \forall j \in \mathbb{Z}_{M} .
\end{aligned}
$$

Note that Eq. (18) corresponds to Eq. (44) with $\left(\alpha_{1}, \alpha_{2}\right)=(0,0)$.

Let us study the modular transformation for the wave function in Eq. (44). First, we check the consistency of the boundary conditions under the modular transformation. For example, the wave functions after the $T$ transformation satisfy

\footnotetext{
${ }^{5}$ The wave function on the magnetized $T^{2} \simeq \mathbb{C} / \Lambda$ with the $\mathbb{Z}_{N}$ SS phases is related to the $\mathbb{Z}_{N}$-eigenmode wave function on the magnetized $\mathbb{Z}_{N}$ full shifted orbifold of $\tilde{T}^{2} \simeq \mathbb{C} / \tilde{\Lambda}(\tilde{\Lambda}=N \Lambda)$ without SS phases [13,41], as shown in Appendix B. The analyses for the wave functions on the magnetized $T^{2}$ with the $\left(\mathbb{Z}_{N}\right)$ SS phases are consistent with those for the wave functions on the magnetized $\tilde{T}^{2} / \mathbb{Z}_{N}$ full shifted orbifold without SS phases in Ref. [13].
} 
$\psi^{\alpha_{1}^{\prime}, \alpha_{2}^{\prime}}(z+\tau+1, \tau+1)=e^{2 \pi i \alpha_{2}^{\prime}} e^{\pi i M \frac{\operatorname{Im}(\bar{\tau}+1) z}{\operatorname{lm} \tau}} \psi^{\alpha_{1}, \alpha_{2}}(z, \tau+1)$,

while the wave functions before the $T$ transformation satisfy

$\psi^{\alpha_{1}, \alpha_{2}}(z+\tau+1, \tau)=e^{2 \pi i\left(\alpha_{1}+\alpha_{2}-M / 2\right)} e^{\pi i M \frac{\operatorname{Im}(\bar{\tau}+1) z}{\operatorname{Im} \tau}} \psi^{\alpha_{1}, \alpha_{2}}(z, \tau)$.

Thus, in order to see the modular symmetry (particularly the $T$ symmetry) of the wave functions, $\alpha_{2}^{\prime} \equiv \alpha_{1}+\alpha_{2}-$ $M / 2(\bmod 1)$ should be satisfied. Also, $\alpha_{1}^{\prime} \equiv \alpha_{1}(\bmod 1)$ is required under the $T$ transformation. Under the $S$ transformation, similarly, $\alpha_{2}^{\prime} \equiv \alpha_{1}(\bmod 1)$ and $\alpha_{1}^{\prime} \equiv$ $1-\alpha_{2}(\bmod 1)$ are required. Then, the modular transformations in Eqs. (32) and (33) are deformed as

$$
\begin{aligned}
& \psi_{T^{2}}^{\left(j+\alpha_{1}^{\prime}, \alpha_{2}^{\prime}\right), M}(\tilde{\gamma}(z, \tau)) \\
& =\tilde{J}_{1 / 2}(\tilde{\gamma}, \tau) \sum_{k=0}^{M-1} \tilde{\rho}_{T^{2}}(\tilde{\gamma})_{j k} \psi_{T^{2}}^{\left(k+\alpha_{1}, \alpha_{2}\right), M}(z, \tau), \quad \tilde{\gamma} \in \tilde{\Gamma},
\end{aligned}
$$

$\tilde{\rho}_{T^{2}}(\tilde{S})_{j k}=e^{\pi i / 4} \frac{1}{\sqrt{M}} e^{2 \pi i\left((j+1) k+\left(1-\alpha_{1}^{\prime}\right) \alpha_{1}\right) / M} \delta_{\alpha_{2}^{\prime}, \alpha_{1}} \delta_{1-\alpha_{1}^{\prime}, \alpha_{2}}$,

$$
\tilde{\rho}_{T^{2}}(\tilde{T})_{j k}=e^{\pi i\left(j+\alpha_{1}^{\prime}\right)\left(j-\alpha_{1}^{\prime}+x\right) / M} \delta_{j, k} \delta_{\alpha_{1}, \alpha_{1}^{\prime}} \delta_{\alpha_{2}^{\prime}-\alpha_{1}^{\prime}+x / 2, \alpha_{2}},
$$

where $x \equiv M(\bmod 2)$ and $\tilde{\rho}_{T^{2}}(\tilde{\gamma})$ satisfies Eqs. (27)-(30) with $k / 2=1 / 2$, although $\mathbb{I}_{j k}$ in Eq. (27) is modified into $e^{-2 \pi i\left(j+\alpha_{1}^{\prime}\right) / M} \delta_{M-j-1, k} \delta_{1-\alpha_{1}^{\prime}, \alpha_{1}} \delta_{1-\alpha_{2}^{\prime}, \alpha_{2}}$, derived from

$$
\begin{aligned}
\psi_{T^{2}}^{\left(j+\alpha_{1}, \alpha_{2}\right), M}(\tilde{Z}(z, \tau)) & =\psi_{T^{2}}^{\left(j+\alpha_{1}, \alpha_{2}\right), M}(-z, \tau) \\
& =e^{-2 \pi i\left(j+\alpha_{1}\right) / M} \psi_{T^{2}}^{\left(M-\left(j+\alpha_{1}\right), 1-\alpha_{2}\right), M}(z, \tau) .
\end{aligned}
$$

However, Eq. (31) is not obtained in the general SS phases. Note that under the modular transformation, in general, the wave functions with the SS phases $\left(\alpha_{1}, \alpha_{2}\right)$ transform into ones with different SS phases $\left(\alpha_{1}^{\prime}, \alpha_{2}^{\prime}\right)$. Conversely, when $M$ is even, only the wave functions with $\left(\alpha_{1}, \alpha_{2}\right)=(0,0)$ are closed under the modular transformation. This case was reviewed in the previous section. Similarly, when $M$ is odd, only the wave functions with $\left(\alpha_{1}, \alpha_{2}\right)=(1 / 2,1 / 2)$ are closed under the modular transformation. In this case, $\tilde{\rho}_{T^{2}}(\tilde{T})$ satisfies

$$
\tilde{\rho}_{T^{2}}(\tilde{T})^{M}=e^{\pi i / 4} \mathbb{I}, \quad \tilde{\rho}_{T^{2}}(\tilde{T})^{8 M}=\mathbb{I} .
$$

Thus, the wave functions on $T^{2}$ with magnetic flux $M \in$ $2 \mathbb{Z}+1$ and $\mathrm{SS}$ phases $\left(\alpha_{1}, \alpha_{2}\right)=(1 / 2,1 / 2)$ behave as modular forms of weight $1 / 2$. They transform as $M$ dimensional representations, but they can be reducible.

Furthermore, we consider the magnetized $T^{2} / \mathbb{Z}_{2}^{(\mathrm{t})}$ twisted orbifold with SS phases. ${ }^{6}$ In this case, we can only consider the $\mathbb{Z}_{2}$ SS phases, $\left(\alpha_{1}, \alpha_{2}\right)=\left(\ell_{1} / 2, \ell_{2} / 2\right),\left(\ell_{1}, \ell_{2} \in \mathbb{Z}_{2}\right)$, which are derived from

$$
1-\alpha_{1} \equiv \alpha_{1}(\bmod 1), \quad 1-\alpha_{2} \equiv \alpha_{2}(\bmod 1)
$$

The wave functions on the magnetized $T^{2} / \mathbb{Z}_{2}^{(\mathrm{t})}$ twisted orbifold with the $\mathbb{Z}_{2}$ SS phases can be expanded by those on the magnetized $T^{2}$ in Eq. (44) as

$$
\psi_{T^{2} / \mathbb{Z}_{2}^{(\mathrm{t}) m}}^{\left(j+\frac{\ell_{1}}{2}, \frac{\ell_{2}}{2}\right), M}(z, \tau)=\mathcal{N}_{(\mathrm{t})}^{\left(j+\frac{\ell_{1}}{2}, \frac{\ell_{2}}{2}\right)} \sum_{k=0}^{M-1}\left(\delta_{j, k}+(-1)^{m} e^{-2 \pi i\left(j+\frac{\ell_{1}}{2}\right) \ell_{2} / m} \delta_{M-j-\ell_{1}, k}\right) \psi_{T^{2}}^{\left(k+\frac{\ell_{1}}{2}, \frac{\ell_{2}}{2}\right), M}(z, \tau),
$$

where we use Eq. (50) instead of Eq. (34). Then, the modular transformation for the wave functions in Eq. (53) is similarly obtained by replacing Eqs. (48) and (49) with

$$
\tilde{\rho}_{T^{2} / \mathbb{Z}_{2}^{(\mathrm{t}) 0}}(\tilde{S})_{j k}=\mathcal{N}_{(\mathrm{t})}^{\left(j+\frac{\ell_{1}}{2}, \frac{\ell_{2}}{2}\right)} \mathcal{N}_{(\mathrm{t})}^{\left(k+\frac{\ell_{1}}{2}, \frac{\ell_{2}}{2}\right)} \frac{4 e^{\pi i / 4}}{\sqrt{M}} e^{\pi i\left(k \ell_{1}^{\prime}-j \ell_{1}\right)} \cos \left(2 \pi\left(j+\frac{\ell_{1}^{\prime}}{2}\right)\left(k+\frac{\ell_{1}}{2}\right) / M\right) \delta_{\ell_{2}^{\prime}, \ell_{1}} \delta_{\ell_{1}^{\prime}, \ell_{2}},
$$

\footnotetext{
${ }^{6}$ Similarly, the wave functions on the magnetized $T^{2} / \mathbb{Z}_{2}^{(\mathrm{t})}$ twisted orbifold with SS phases are related to those on the magnetized $\tilde{T}^{2} / \mathbb{Z}_{2}$ twisted and full shifted orbifold without SS phases in Ref. [13].
} 


$$
\begin{gathered}
\tilde{\rho}_{T^{2} / \mathbb{Z}_{2}^{(\mathrm{t}) 0}}(\tilde{T})_{j k}=e^{\pi i\left(j+\frac{\ell_{1}^{\prime}}{2}\right)\left(j-\frac{\ell_{1}^{\prime}}{2}+x\right) / M} \delta_{j, k} \delta_{\ell_{1}^{\prime}, \ell_{1}} \delta_{\ell_{2}^{\prime}-\ell_{1}^{\prime}+x, \ell_{2}}, \\
\tilde{\rho}_{T^{2} / \mathbb{Z}_{2}^{(\mathrm{t}) 1}}(\tilde{S})_{j k}=\mathcal{N}_{(\mathrm{t})}^{\left(j+\frac{\ell_{1}}{2}, \frac{\ell_{2}}{2}\right)} \mathcal{N}_{(\mathrm{t})}^{\left(k+\frac{\ell_{1}}{2}, \frac{\ell_{2}}{2}\right)} \frac{4 i e^{\pi i / 4}}{\sqrt{M}} e^{\pi i\left(k \ell_{1}^{\prime}-j \ell_{1}\right)} \sin \left(2 \pi\left(j+\frac{\ell_{1}^{\prime}}{2}\right)\left(k+\frac{\ell_{1}}{2}\right) / M\right) \delta_{\ell_{2}^{\prime}, \ell_{1}} \delta_{\ell_{1}^{\prime}, \ell_{2}}, \\
\tilde{\rho}_{T^{2} / \mathbb{Z}_{2}^{(\mathrm{t}) 1}}(\tilde{T})_{j k}=e^{\pi i\left(j+\frac{\ell_{1}^{\prime}}{2}\right)\left(j-\frac{\ell_{1}^{\prime}}{2}+x\right) / M} \delta_{j, k} \delta_{\ell_{1}^{\prime}, \ell_{1}} \delta_{\ell_{2}^{\prime}-\ell_{1}^{\prime}+x, \ell_{2}} .
\end{gathered}
$$

In particular, when $M=$ even and $\left(\alpha_{1}, \alpha_{2}\right)=(0,0)$, they correspond to Eqs. (40) and (41). When $M=$ odd and $\left(\alpha_{1}, \alpha_{2}\right)=(1 / 2,1 / 2)$, they become

$$
\begin{aligned}
& \tilde{\rho}_{T^{2} / \mathbb{Z}_{2}^{(\mathrm{t})}(\tilde{S})_{j k}}=\mathcal{N}_{(\mathrm{t})}^{\left(j+\frac{1}{2}, \frac{1}{2}\right)} \mathcal{N}_{(\mathrm{t})}^{\left(k+\frac{1}{2}, \frac{1}{2}\right)} \frac{4 e^{\pi i / 4}}{\sqrt{M}} e^{\pi i(k-j)} \cos \left(2 \pi\left(j+\frac{1}{2}\right)\left(k+\frac{1}{2}\right) / M\right), \\
& \tilde{\rho}_{T^{2} / \mathbb{Z}_{2}^{(t) 0}}(\tilde{T})_{j k}=e^{\pi i\left(j+\frac{1}{2}\right)^{2} / M} \delta_{j, k}, \\
& \tilde{\rho}_{T^{2} / \mathbb{Z}_{2}^{(\mathrm{t}) 1}}(\tilde{S})_{j k}=\mathcal{N}_{(\mathrm{t})}^{\left(j+\frac{1}{2}, \frac{1}{2}\right)} \mathcal{N}_{(\mathrm{t})}^{\left(k+\frac{1}{2}, \frac{1}{2}\right)} \frac{4 i e^{\pi i / 4}}{\sqrt{M}} e^{\pi i(k-j)} \sin \left(2 \pi\left(j+\frac{1}{2}\right)\left(k+\frac{1}{2}\right) / M\right),
\end{aligned}
$$

$$
\tilde{\rho}_{T^{2} / \mathbb{Z}_{2}^{(t) 1}}(\tilde{T})_{j k}=e^{\pi i\left(j+\frac{1}{2}\right)^{2} / M} \delta_{j, k}
$$

where Eqs. (58)-(61) for each $m \in \mathbb{Z}_{2}$ satisfy Eqs. (27)(30), and Eq. (51) with $k / 2=1 / 2$, although $\mathbb{I}_{j k}=\delta_{j, k}$ in Eq. (27) is modified into $(-1)^{m} \delta_{j, k}$, derived from Eq. (37). Note that there are $(M-1) / 2 \mathbb{Z}_{2}^{(\mathrm{t})}$-even $(m=0)$ modes and $(M+1) / 2 \mathbb{Z}_{2}^{(\mathrm{t})}$-odd $(m=1)$ modes when $M=$ odd and $\left(\alpha_{1}, \alpha_{2}\right)=(1 / 2,1 / 2)$. Thus, both $\mathbb{Z}_{2}^{(\mathrm{t})}$-even and -odd

TABLE I. Number of $\mathbb{Z}_{2}^{(\mathrm{t})}$-even $(m=0)$ modes, $N_{0}(M)$, and $\mathbb{Z}_{2}^{(\mathrm{t})}$-odd $(m=1)$ modes, $N_{1}(M)$, on the $T^{2} / \mathbb{Z}_{2}^{(\mathrm{t})}$ twisted orbifold with $M=$ even and $\left(\alpha_{1}, \alpha_{2}\right)=(0,0)$, and the order of $\tilde{T}$. The three generations are boxed.

\begin{tabular}{lccccc}
\hline \hline & $M$ & 2 & 4 & 6 & 8 \\
\hline $\mathbb{Z}_{2}^{(\mathrm{t})}$-even: $N_{0}(M)$ & $\frac{M}{2}+1$ & 2 & 3 & 4 & 5 \\
$\mathbb{Z}_{2}^{(\mathrm{t})}$-odd: $N_{1}(M)$ & $\frac{M}{2}-1$ & 0 & 1 & 2 & 3 \\
order $h$ of $\tilde{T}\left(T^{h}=\mathbb{I}\right)$ & $2 M$ & 4 & 8 & 12 & 16 \\
\hline \hline
\end{tabular}

TABLE II. Number of $\mathbb{Z}_{2}^{(\mathrm{t})}$-even $(m=0)$ modes, $N_{0}(M)$, and $\mathbb{Z}_{2}^{(\mathrm{t})}$-odd $(m=1)$ modes, $N_{1}(M)$, on the $T^{2} / \mathbb{Z}_{2}^{(\mathrm{t})}$ twisted orbifold with $M=$ odd and $\left(\alpha_{1}, \alpha_{2}\right)=(1 / 2,1 / 2)$, and the order of $\tilde{T}$. The three generations are boxed.

\begin{tabular}{lccccc}
\hline \hline & $M$ & 1 & 3 & 5 & 7 \\
\hline $\mathbb{Z}_{2}^{(\mathrm{t})}$-even: $N_{0}(M)$ & $\frac{M-1}{2}$ & 0 & 1 & 2 & 3 \\
$\mathbb{Z}_{2}^{(\mathrm{t})}$-odd: $N_{1}(M)$ & $\frac{M+1}{2}$ & 1 & 2 & 3 & 4 \\
order $h$ of $\tilde{T}\left(T^{h}=\mathbb{I}\right)$ & $8 M$ & 8 & 24 & 40 & 56 \\
\hline \hline
\end{tabular}

mode wave functions on the $T^{2} / \mathbb{Z}_{2}^{(\mathrm{t})}$ twisted orbifold with magnetic flux $M \in 2 \mathbb{Z}+1$ and the SS phases $\left(\alpha_{1}, \alpha_{2}\right)=$ $(1 / 2,1 / 2)$ behave as modular forms of weight $1 / 2$. Then, they transform as $(M-1) / 2$ - and $(M+1) / 2$-dimensional representations for $\mathbb{Z}_{2}^{(\mathrm{t})}$-even and -odd modes, respectively. We show the number of $\mathbb{Z}_{2}^{(\mathrm{t})}$ eigenmodes, $N_{m}(M)$, which have the modular symmetry, and the order of $\tilde{T}$, i.e., $\tilde{T}^{h}=\mathbb{I}$ in Tables I and II.

\section{MODULAR FLAVOR GROUPS OF THREE- GENERATION MODES ON MAGNETIZED $T^{2} / \mathbb{Z}_{2}^{(\mathrm{t})}$ TWISTED ORBIFOLDS}

As mentioned in the Introduction, in Ref. [29] threedimensional representations were obtained from specific finite modular subgroups: $\Gamma_{3} \simeq A_{4}, \quad \Gamma_{4} \simeq S_{4}, \quad \Gamma_{5} \simeq A_{5}$, $\Gamma_{7} \simeq \operatorname{PSL}\left(2, \mathbb{Z}_{7}\right), \Gamma_{8} \supset \Delta(96)$, and $\Gamma_{16} \supset \Delta(384) .{ }^{7}$ In this section, we show that the three-generation modes on the magnetized $T^{2} / \mathbb{Z}_{2}^{(\mathrm{t})}$ twisted orbifold shown in Tables I and II are the representations of the corresponding covering or central extended groups of the modular flavor groups.

\section{A. $T^{2} / \mathbb{Z}_{2}^{(t)}$ twisted orbifold with magnetic flux $M=$ even and vanishing Scherk-Schwarz phases}

In this subsection we show the modular flavor groups of the three-generation modes on the $T^{2} / \mathbb{Z}_{2}^{(\mathrm{t})}$ twisted orbifold with $M=$ even and $\left(\alpha_{1}, \alpha_{2}\right)=(0,0)$. As shown in Table I,

\footnotetext{
${ }^{7}$ See Refs. [2,3,42] for the algebraic relations for the generators of each non-Abelian discrete flavor group.
} 
the three-generation modes are obtained from the $\mathbb{Z}_{2}^{(\mathrm{t})}$-even modes with $M=4$ and the $\mathbb{Z}_{2}^{(\mathrm{t})}$-odd modes with $M=8$. In the following, we show that they are the representations of $\tilde{\Delta}(96)$ and $\tilde{\Delta}(384)$, which are subgroups of $\tilde{\Gamma}_{8}$ and $\tilde{\Gamma}_{16}$, respectively, and are the quadruple covering groups of $\Delta(96)$ and $\Delta(384)$, respectively.

First, $\Gamma_{N}$ satisfy

$$
S^{2}=(S T)^{3}=T^{N}=\mathbf{1} .
$$

On the other hand, $\Delta(96) \simeq\left(Z_{4} \times Z_{4}^{\prime}\right) \rtimes Z_{3} \rtimes Z_{2} \simeq \Delta(48) \rtimes Z_{2}$ and $\Delta(384) \simeq\left(Z_{8} \times Z_{8}^{\prime}\right) \rtimes Z_{3} \rtimes Z_{2} \simeq \Delta(192) \rtimes Z_{2}$ satisfy

$$
\begin{aligned}
a^{M} & =a^{M}=b^{3}=c^{2}=\mathbf{1}, \quad(M=4,8), \\
a a^{\prime} & =a^{\prime} a, \quad c b c^{-1}=b^{-1}, \quad b a b^{-1}=a^{-1} a^{\prime-1}, \\
b a^{\prime} b^{-1} & =a, \quad c a c^{-1}=a^{-1}, \quad c a^{\prime} c^{-1}=a^{-1},
\end{aligned}
$$

where $a^{\left({ }^{\prime}\right)}, b, c$ denote the generators of $Z_{M}^{(\prime)}(M=4,8), Z_{3}$, $Z_{2}$, respectively $[2,3,42]$. In order to obtain $\Delta(96)$ and $\Delta(384)$ from the above algebra (62) for $N=8$ and 16 , respectively, the relation

$$
\left(S^{-1} T^{-1} S T\right)^{3}=\mathbf{1}
$$

should also be satisfied. Actually, we can show that if $S$ and $T$ satisfy Eq. (64) in addition to Eq. (62) for $N=2 M$, $M \in 4 \mathbb{Z}$, the generators ${ }^{8}$

$$
\begin{aligned}
& a=S T^{2} S T^{4}, \quad a^{\prime}=S T^{2} S^{-1} T^{-2}, \\
& b=T^{\frac{M}{2}+3} S T^{M}, \quad c=S T^{M-2} S T^{\frac{3}{2} M-1}
\end{aligned}
$$

satisfy Eq. (63) in Appendix C (see also Refs. [29,43]). In other words, we can obtain $\Delta\left(6 M^{2}\right)$ from $\Gamma_{2 M}$ by satisfying the additional relation in Eq. (64). Similarly, $\tilde{\Gamma}_{2 M}$ satisfy Eqs. (27)-(31) with $k / 2=1 / 2$ and $N=2 M$. If Eq. (64) is also satisfied, especially for $M \in 4 \mathbb{Z}$, the generators

$$
\begin{aligned}
& a=S T^{2} S^{5} T^{4}, \quad a^{\prime}=S T^{2} S^{-1} T^{-2}, \\
& b=T^{\frac{M}{2}+3} S^{\frac{3}{2} M-1} T^{M}, \quad c=S T^{M-2} S T^{\frac{3}{2} M-1}
\end{aligned}
$$

satisfy

$$
\begin{aligned}
a^{M} & =a^{M}=b^{3}=c^{8}=\mathbf{1}, \quad a a^{\prime}=a^{\prime} a, \\
c b c^{-1} & =b^{-1}, \quad b a b^{-1}=a^{-1} a^{\prime-1}, \\
b a^{\prime} b^{-1} & =a, \quad c a c^{-1}=a^{-1}, \quad c a^{\prime} c=a^{-1},
\end{aligned}
$$

\footnotetext{
${ }^{8}$ For $N=2 M, M=2(2 s-1) s \in \mathbb{Z}$, similarly, the generators $a=S T^{2} S T^{4}, a^{\prime}=S T^{2} S^{-1} T^{-2}, b=T^{\frac{M}{2}} S T^{M}$, and $c=S T^{M} S T^{\frac{3}{2} M}$ satisfy Eq. (63).
}

which means that the generators in Eq. (66) are those of $\tilde{\Delta}\left(6 M^{2}\right) \simeq\left(Z_{M} \times Z_{M}\right) \rtimes Z_{3} \rtimes Z_{8} \simeq \Delta\left(3 M^{2}\right) \rtimes Z_{8}$, where $a^{\left({ }^{\prime}\right)}$, $b, c$ denote those of $Z_{M}^{()}, Z_{3}, Z_{8}$, respectively. (We give the proof in Appendix C.) In other words, we can obtain $\tilde{\Delta}\left(6 M^{2}\right)$, especially for $M \in 4 \mathbb{Z}$, from $\tilde{\Gamma}_{2 M}$ by satisfying the additional relation in Eq. (64).

Let us study the case of the three-generation modes on the $T^{2} / \mathbb{Z}_{2}^{(\mathrm{t})}$ twisted orbifold with $M=4,8$ and $\left(\alpha_{1}, \alpha_{2}\right)=(0,0)$. The $S$ and $T$ transformation matrices for the $\mathbb{Z}_{2}^{(\mathrm{t})}$-even modes with $M=4$ are given by

$$
S=\frac{e^{\pi i / 4}}{2}\left(\begin{array}{ccc}
1 & \sqrt{2} & 1 \\
\sqrt{2} & 0 & -\sqrt{2} \\
1 & -\sqrt{2} & 1
\end{array}\right), \quad T=\left(\begin{array}{lll}
1 & & \\
& e^{\pi i / 4} & \\
& & -1
\end{array}\right)
$$

and those for the $\mathbb{Z}_{2}^{(\mathrm{t})}$-odd modes with $M=8$ are given by

$S=\frac{e^{3 \pi i / 4}}{2}\left(\begin{array}{ccc}1 & \sqrt{2} & 1 \\ \sqrt{2} & 0 & -\sqrt{2} \\ 1 & -\sqrt{2} & 1\end{array}\right), T=e^{\pi i / 8}\left(\begin{array}{ccc}1 & & \\ & e^{3 \pi i / 8} & \\ & & -1\end{array}\right)$.

Note that here and hereafter (as well as in Sec. VI) we omit $\rho$. The above $S$ and $T$ matrices in both of Eqs. (68) and (69) can be written by

$$
\begin{aligned}
& S=\frac{e^{i \theta_{1}}}{2}\left(\begin{array}{ccc}
1 & \sqrt{2} & 1 \\
\sqrt{2} & 0 & -\sqrt{2} \\
1 & -\sqrt{2} & 1
\end{array}\right), \\
& T=e^{i \theta_{2}}\left(\begin{array}{ccc}
1 & \\
& e^{i \theta_{3}} & \\
& & -1
\end{array}\right), \forall \theta_{1,2,3} \in \mathbb{R},
\end{aligned}
$$

and we can check that Eq. (70) satisfies Eq. (64) in general. Thus, the three-generation $\mathbb{Z}_{2}^{(\mathrm{t})}$-even modes with $M=4$ and $\mathbb{Z}_{2}^{(\mathrm{t})}$-odd modes with $M=8$ are transformed under the modular transformation as the three-dimensional representations of $\tilde{\Delta}(96)$ and $\tilde{\Delta}(384)$, respectively. ${ }^{9}$

We also comment on the modular flavor anomaly. As discussed in Refs. [22,44], the transformation $g$ can be anomalous if $\operatorname{det}(g) \neq 1$. Then, let us see the anomaly of the modular flavor group $\tilde{\Delta}\left(6 M^{2}\right)$. From Eqs. (27)-(31) with $k / 2=1 / 2$ and $N=2 M$, and Eqs. (66) and (67), we can obtain

\footnotetext{
${ }^{9}$ See also Ref. [11].
} 


$$
\begin{aligned}
& \operatorname{det}(a)=\operatorname{det}\left(a^{\prime}\right)=\operatorname{det}(b)=1, \\
& \operatorname{det}(c)=\operatorname{det}(T)^{\frac{M}{2}+3}, \quad \operatorname{det}(c)^{8}=1 .
\end{aligned}
$$

Actually, Eqs. (68) and (69) both satisfy Eq. (71) and $\operatorname{det}(c)=e^{\pi i / 4}$. Thus, only $Z_{8}$ symmetry, generated by $c$, can be anomalous, ${ }^{10}$ and then $\Delta(48)$ and $\Delta(192)$ remain anomaly free, respectively.

\section{B. $T^{2} / \mathbb{Z}_{2}^{(\mathrm{t})}$ twisted orbifold with magnetic flux $M=$ odd and the Scherk-Schwarz phases $\left(\alpha_{1}, \alpha_{2}\right)=(1 / 2,1 / 2)$}

In this subsection we show the modular flavor groups of the three-generation modes on the $T^{2} / \mathbb{Z}_{2}^{(\mathrm{t})}$ twisted orbifold with $M=$ odd and $\left(\alpha_{1}, \alpha_{2}\right)=(1 / 2,1 / 2)$. As shown in Table II, the three-generation modes are obtained from the $\mathbb{Z}_{2}^{(\mathrm{t})}$-odd modes with $M=5$ and the $\mathbb{Z}_{2}^{(\mathrm{t})}$-even modes with $M=7$.

First, the $S$ and $T$ transformation matrices for the $\mathbb{Z}_{2}^{(\mathrm{t})}$-odd modes with $M=5$ are given by

$$
\begin{aligned}
& S=\frac{i e^{\pi i / 4}}{\sqrt{5}}\left(\begin{array}{ccc}
2 \sin \left(\frac{\pi}{10}\right) & 2 e^{\pi i / 5} \sin \left(\frac{3 \pi}{10}\right) & \sqrt{2} e^{2 \pi i / 5} \\
2 e^{-\pi i / 5} \sin \left(\frac{3 \pi}{10}\right) & 2 \sin \left(\frac{\pi}{10}\right) & -\sqrt{2} e^{\pi i / 5} \\
\sqrt{2} e^{-2 \pi i / 5} & -\sqrt{2} e^{-\pi i / 5} & 1
\end{array}\right) \\
& T=\left(\begin{array}{ccc}
e^{\pi i / 20} & \\
& e^{9 \pi i / 20} & \\
& e^{25 \pi i / 20}
\end{array}\right)
\end{aligned}
$$

which satisfy Eqs. (27)-(30) and (51) with $k / 2=1 / 2$ and replacing $\mathbb{I}$ in Eq. (27) with $(-1)^{m=1} \mathbb{I}=-\mathbb{I}$. When we define the generators

$$
a=S T^{5}, \quad b=S T^{21}, \quad c=T^{5}
$$

from the above $S$ and $T$ in Eq. (72), they satisfy

$$
a^{2}=b^{3}=(a b)^{5}=c^{8}=\mathbf{1}, \quad a c=c a, \quad b c=c b,
$$

which means that they are the generators of $A_{5} \times Z_{8}$. Thus, the three-generational $\mathbb{Z}_{2}^{(\mathrm{t})}$-odd modes with $M=5$ are transformed under the modular transformation as the three-dimensional representations of $A_{5} \times Z_{8}$.

Next, the $S$ and $T$ transformation matrices for the $\mathbb{Z}_{2}^{(\mathrm{t})}$-even modes with $M=7$ are given by

\footnotetext{
${ }^{10}$ The anomalous symmetry, which is the discrete subsymmetry of $U(1)$, can be canceled by the Green-Schwarz mechanism.
}

$S=\frac{2 e^{\pi i / 4}}{\sqrt{7}}\left(\begin{array}{ccc}\cos \left(\frac{\pi}{14}\right) & e^{\pi i / 7} \cos \left(\frac{3 \pi}{14}\right) & e^{2 \pi i / 7} \cos \left(\frac{5 \pi}{14}\right) \\ e^{-\pi i / 7} \cos \left(\frac{3 \pi}{14}\right) & \cos \left(\frac{9 \pi}{14}\right) & -e^{\pi i / 7} \cos \left(\frac{\pi}{14}\right) \\ e^{-2 \pi i / 7} \cos \left(\frac{5 \pi}{14}\right) & -e^{-\pi i / 7} \cos \left(\frac{\pi}{14}\right) & \cos \left(\frac{3 \pi}{14}\right)\end{array}\right)$,

$T=\left(\begin{array}{lll}e^{\pi i / 28} & & \\ & e^{9 \pi i / 28} & \\ & & e^{25 \pi i / 28}\end{array}\right)$

which satisfy Eqs. (27)-(30) and (51) with $k / 2=1 / 2$. They also satisfy

$$
\left(S^{-1} T^{-1} S T\right)^{4}=\mathbf{1}
$$

When we define the generators

$$
a=S T^{21}, \quad b=S^{7} T^{3}, \quad c=T^{7}
$$

from the above $S$ and $T$ in Eq. (75), they satisfy

$a^{2}=b^{4}=(a b)^{7}=\left(a^{-1} b^{-1} a b\right)^{4}=c^{8}=\mathbf{1}$,

$a c=c a, \quad b c=c b$,

which means that they are the generators of $\operatorname{PSL}\left(2, Z_{7}\right) \times Z_{8}$. Thus, the three-generational $\mathbb{Z}_{2}^{(\mathrm{t})}$-even modes with $M=7$ are transformed under the modular transformation as the three-dimensional representations of $\operatorname{PSL}\left(2, Z_{7}\right) \times Z_{8}$.

Similarly, we comment on the anomaly of these modular flavor groups. From Eqs. (27)-(30) with $k / 2=1 / 2$, and Eqs. (51), (73), and (74) as well as Eqs. (77) and (78), we can obtain

$\operatorname{det}(a)=\operatorname{det}(b)=1, \quad \operatorname{det}(c)=\operatorname{det}\left(e^{\pi i / 4} \mathbb{I}\right), \quad \operatorname{det}(c)^{8}=1$.

Actually, Eqs. (72) and (75) satisfy Eq. (79). Thus, in both cases, only $Z_{8}$ symmetry (generated by $c$ ) can be anomalous, and then $A_{5}$ and $\operatorname{PSL}\left(2, Z_{7}\right)$ remain anomaly free.

\section{MODULAR SYMMETRY ON MAGNETIZED ORBIFOLDS OF $T^{2} \times T^{2}$}

In this section we extend the analyses to the modular symmetry on magnetized orbifolds of $T_{1}^{2} \times T_{2}^{2}$, where both of the moduli on $T_{i}^{2}(i=1,2), \tau_{i}$, are identified each other, i.e., $\tau_{1}=\tau_{2} \equiv \tau$ (see Ref. [14]). First, let us consider the modular transformation for the wave functions on the $T_{1}^{2} / \mathbb{Z}_{2}^{\left(\mathrm{t}_{1}\right)} \times T^{2} / \mathbb{Z}_{2}^{\left(\mathrm{t}_{2}\right)}$ with magnetic flux $M^{(i)}=$ even and the SS phases $\left(\alpha_{1}^{(i)}, \alpha_{2}^{(i)}\right)=(0,0)$, and magnetic flux $M^{(i)}=$ odd and the SS phases $\left(\alpha_{1}^{(i)}, \alpha_{2}^{(i)}\right)=(1 / 2,1 / 2)$ on each $T_{i}^{2} / \mathbb{Z}_{2}^{\left(\mathrm{t}_{i}\right)}$. The wave functions transform under the modular transformation as 


$$
\begin{aligned}
& \Psi_{\left(\mathrm{t}_{1}\right) m_{1}\left(\mathrm{t}_{2}\right) m_{2}}^{j^{(1)} \boldsymbol{j}^{(2)}, M^{(1)} M^{(2)}}\left(\gamma\left(z_{1}, z_{2}, \tau\right)\right)=J_{1}(\gamma, \tau) \sum_{k^{(1)}=0}^{N_{m_{1}}\left(M^{(1)}\right)} \sum_{k^{(2)}=0}^{N_{m_{2}}\left(M^{(2)}\right)} \rho_{\left(\mathrm{t}_{1}\right) m_{1}\left(\mathrm{t}_{2}\right) m_{2}}(\gamma)_{\left(j^{(1)} j^{(2)}\right)\left(k^{(1)} k^{(2)}\right)} \Psi_{\left.\left(\mathrm{t}_{1}\right) m_{1}, \mathrm{t}_{2}\right) m_{2}}^{k^{(1)} k^{(2)} M^{(2)}}\left(z_{1}, z_{2}, \tau\right) \\
& m_{1} \in \mathbb{Z}_{2}^{\left(\mathrm{t}_{1}\right)}, \quad m_{2} \in \mathbb{Z}_{2}^{\left(\mathrm{t}_{2}\right)}, \quad \gamma \in \Gamma, \\
& \Psi_{\left(\mathrm{t}_{1}\right) m_{1}\left(\mathrm{t}_{2}\right) m_{2}}^{j^{(1)} j^{(2)}, M^{(1)} M^{(2)}}\left(z_{1}, z_{2}, \tau\right)=\psi_{T_{1}^{2} / \mathbb{Z}_{2}^{\left(\mathrm{t}_{1}\right) m_{1}}}^{\left(k_{2}^{(1)}+\alpha_{1}^{(1)}, \alpha^{(1)}\right), M^{(1)}}\left(z_{1}, \tau\right) \psi_{T_{2}^{2} / \mathbb{Z}_{2}^{\left(\mathrm{t}_{2}\right) m_{2}}}^{\left(k_{2}^{(2)}+\alpha^{(2)}, \alpha^{(2)}\right), M^{(2)}}\left(z_{2}, \tau\right), \\
& \rho_{\left(\mathrm{t}_{1}\right) m_{1}\left(\mathrm{t}_{2}\right) m_{2}}(S)_{\left(j^{(1)} j^{(2)}\right)\left(k^{(1)} k^{(2)}\right)}=\tilde{\rho}_{T_{1}^{2} / \mathbb{Z}_{2}^{\left(\mathrm{t}_{1}\right) m_{1}}}(\tilde{S})_{j^{(1)} k^{(1)}} \tilde{\rho}_{T_{2}^{2} / \mathbb{Z}_{2}^{\left(\mathrm{t}_{2}\right) m_{2}}}(\tilde{S})_{j^{(2)} k^{(2)}}, \\
& \rho_{\left(\mathrm{t}_{1}\right) m_{1}\left(\mathrm{t}_{2}\right) m_{2}}(T)_{\left(j^{(1)} j^{(2)}\right)\left(k^{(1)} k^{(2)}\right)}=\tilde{\rho}_{T_{1}^{2} / \mathbb{Z}_{2}^{\left(\mathrm{t}_{1}\right) m_{1}}}(\tilde{T})_{j^{(1)} k^{(1)}} \tilde{\rho}_{T_{2}^{2} / \mathbb{Z}_{2}^{\left(\mathrm{t}_{2}\right) m_{2}}}(\tilde{T})_{j^{(2)} k^{(2)}},
\end{aligned}
$$

where $\tilde{\rho}_{T_{2}^{2} / \mathbb{Z}_{2}^{\left(t_{i}\right) m_{i}}}(\tilde{\gamma})(i=1,2)$ correspond to Eqs. (40)-(41) for $M^{(i)}=$ even and $\left(\alpha_{1}^{(i)}, \alpha_{2}^{(i)}\right)=(0,0)$ or Eqs. (58)-(61) for $M^{(i)}=$ odd and $\left(\alpha_{1}^{(i)}, \alpha_{2}^{(i)}\right)=(1 / 2,1 / 2)$. Then, $\rho_{\left(\mathrm{t}_{1}\right) m_{1}\left(\mathrm{t}_{2}\right) m_{2}}(\gamma)$ satisfies Eq. (8) with $k=1$, where $\rho(Z)=-\mathbb{I}$ is replaced by $\rho_{\left(\mathrm{t}_{1}\right) m_{1}\left(\mathrm{t}_{2}\right) m_{2}}(Z)=-(-1)^{m_{1}+m_{2}} \mathbb{I}$, and also satisfies ${ }^{11}$

$$
\begin{aligned}
& \rho(T)^{2 \operatorname{lcm}\left(M^{(1)}, M^{(2)}\right)}=\mathbb{I}, \quad\left(M^{(1)}=2 s^{(1)}, M^{(2)}=2 s^{(2)}\right), \\
& \rho(T)^{2 \mathrm{lcm}\left(M^{(1)}, M^{(2)}\right)}=\mathbb{I}, \quad\left(M^{(1)}=4 s^{(1)}, M^{(2)}=2 s^{(2)}-1\right), \\
& \rho(T)^{2 \mathrm{lcm}\left(M^{(1)}, M^{(2)}\right)}=-\mathbb{I}, \quad \rho(T)^{4 \operatorname{lcm}\left(M^{(1)}, M^{(2)}\right)}=\mathbb{I}, \quad\left(M^{(1)}=2\left(2 s^{(1)}-1\right), M^{(2)}=2 s^{(2)}-1\right), \\
& \rho(T)^{\operatorname{lcm}\left(M^{(1)}, M^{(2)}\right)}=e^{\pi i \frac{M^{(1)}+M^{(2)}}{4 \operatorname{gcd}\left(M^{(1)}, M^{(2)}\right)} \mathbb{I}}, \quad\left(M^{(1)}=2 s^{(1)}-1, M^{(2)}=2 s^{(2)}-1\right), \\
& \Rightarrow \rho(T)^{N}=\mathbb{I}, \quad N= \begin{cases}\operatorname{lcm}\left(M^{(1)}, M^{(2)}\right) & \left(M^{(1)}+M^{(2)} \in 8 \mathbb{Z}\right), \\
2 \operatorname{lcm}\left(M^{(1)}, M^{(2)}\right) & \left(M^{(1)}+M^{(2)} \in 4 \mathbb{Z}\right), \\
4 \operatorname{lcm}\left(M^{(1)}, M^{(2)}\right) & \left(M^{(1)}+M^{(2)} \in 2 \mathbb{Z}\right),\end{cases}
\end{aligned}
$$

corresponding to Eq. (9), where $s^{(1)}, s^{(2)} \in \mathbb{Z}$ and we omit the $\mathbb{Z}_{2}^{(\mathrm{t})}$ indices since the above relations are independent of them. Thus, the wave functions on the magnetized $T_{1}^{2} / \mathbb{Z}_{2}^{\left(\mathrm{t}_{1}\right)} \times T^{2} / \mathbb{Z}_{2}^{\left(\mathrm{t}_{2}\right)}$ orbifold behave as modular forms of weight 1 , and then they transform as $N_{m_{1}}\left(M^{(1)}\right) N_{m_{2}}\left(M^{(2)}\right)$-dimensional representations, where $N_{m_{i}}\left(M^{(i)}\right)(i=1,2)$ denote the number of zero-mode wave functions on $T_{i}^{2} / \mathbb{Z}_{2}^{\left(\mathrm{t}_{i}\right)}$. These can be irreducible representations. We will study their flavor symmetries in the next section. Also, note that when $m_{1}+m_{2}=1, S^{2}=\mathbb{I}$ is satisfied even though the modular weight $k=1{ }^{12}$

We can further consider the $\mathbb{Z}_{2}^{(\mathrm{p})}$ permutation orbifold if $M^{(1)}=M^{(2)}=M, \alpha_{i}^{(1)}=\alpha_{i}^{(2)}=\alpha_{i}(i=1,2)$, and $m_{1}=m_{2}=m$. The $\mathbb{Z}_{2}^{(\mathrm{p})}$ permutation means that the transformation of the complex coordinate of $T_{1}^{2} / \mathbb{Z}_{2}^{\left(\mathrm{t}_{1}\right)} \times T^{2} / \mathbb{Z}_{2}^{\left(\mathrm{t}_{2}\right)}$ : $\left(z_{1}, z_{2}\right) \rightarrow\left(z_{2}, z_{1}\right)$, and then the $\mathbb{Z}_{2}^{(\mathrm{p})}$ permutation orbifold can be considered by identifying $z_{1}$ and $z_{2}$. Hence, the wave functions on the $\mathbb{Z}_{2}^{(\mathrm{p})}$ permutation orbifold of $T_{1}^{2} / \mathbb{Z}_{2}^{\left(\mathrm{t}_{1}\right)} \times T^{2} / \mathbb{Z}_{2}^{\left(\mathrm{t}_{2}\right)}$, i.e., the $\left(T_{1}^{2} \times T_{2}^{2}\right) /\left(\mathbb{Z}_{2}^{(\mathrm{t})} \times \mathbb{Z}_{2}^{(\mathrm{p})}\right)$ orbifold, are expressed as

$$
\begin{aligned}
\Psi_{(t) m(p) n}^{j^{(1)} j^{(2)}, M}\left(z_{1}, z_{2}, \tau\right) & =\mathcal{N}_{(\mathrm{t}, \mathrm{p})}^{j^{(1)} j^{(2)}}\left(\Psi_{(\mathrm{t}) m(\mathrm{t}) m}^{j^{(1)} j^{(2)}, M M}\left(z_{1}, z_{2}, \tau\right)+(-1)^{n} \Psi_{(\mathrm{t}) m(\mathrm{t}) m}^{j^{(1)} j^{(2)}, M M}\left(z_{2}, z_{1}, \tau\right)\right), \\
m & \in \mathbb{Z}_{2}^{(\mathrm{t})}, \quad n \in \mathbb{Z}_{2}^{(\mathrm{p})}, \quad j^{(1)} \geq j^{(2)}, \quad \mathcal{N}_{(\mathrm{t}, \mathrm{p})}^{j^{(1)} j^{(2)}}= \begin{cases}1 / 2 & \left(j^{(1)}=j^{(2)}\right), \\
1 / \sqrt{2} & \left(j^{(1)}>j^{(2)}\right),\end{cases}
\end{aligned}
$$

and they satisfy the boundary condition

\footnotetext{
${ }^{11} \operatorname{lcm}(a, b)$ denotes the least common multiple of $a$ and $b$, and $\operatorname{gcd}(a, b)$ denotes the greatest common divisor of $a$ and $b$.

${ }^{12}$ This situation does not appear in modular forms, and actually the wave functions vanish at $z_{1}=z_{2}=0$.
} 
TABLE III. Number of ( $\mathbb{Z}_{2}^{(\mathrm{t})}$ twist, $\mathbb{Z}_{2}^{(\mathrm{p})}$ permutation) eigenmodes, $N_{(m, n)}(M)$, on the $\left(T_{1}^{2} \times T_{2}^{2}\right) /\left(\mathbb{Z}_{2}^{(\mathrm{t})} \times \mathbb{Z}_{2}^{(\mathrm{p})}\right)$ orbifold with $M=$ even and $\left(\alpha_{1}, \alpha_{2}\right)=(0,0)$, and the order of $T$. The three generations are boxed.

\begin{tabular}{lccccc}
\hline \hline & $M$ & 2 & 4 & 6 & 8 \\
\hline (even, even): $N_{(0,0)}(M)$ & $(M+2)(M+4) / 8$ & 3 & 6 & 10 & 15 \\
(even, odd): $N_{(0,1)}(M)$ & $M(M+2) / 8$ & 1 & 3 & 6 & 10 \\
(odd, even): $N_{(1,0)}(M)$ & $M(M-2) / 8$ & 0 & 1 & 3 & 6 \\
(odd, odd): $N_{(1,1)}(M)$ & $(M-2)(M-4) / 8$ & 0 & 0 & 1 & 3 \\
order $h$ of $T\left(T^{h}=\mathbb{I}\right)$ & $2 M$ & 4 & 8 & 12 & 16 \\
\hline \hline
\end{tabular}

$$
\Psi_{(t) m(p) n}^{j^{(1)} j^{(2)}, M}\left(z_{2}, z_{1}, \tau\right)=(-1)^{n} \Psi_{(t) m(p) n}^{j^{(1)} j^{(2)}, M}\left(z_{1}, z_{2}, \tau\right)
$$

in addition to those in Eqs. (15), (16), and (37). Thus, we can obtain $N_{m}(M)\left(N_{m}(M)+1\right) / 2 \mathbb{Z}_{2}^{(\mathrm{p})}$-even $(n=0)$ modes and $N_{m}(M)\left(N_{m}(M)-1\right) / 2 \mathbb{Z}_{2}^{(\mathrm{p})}$-odd $(n=1)$ modes. We show the number of $\left(\mathbb{Z}_{2}^{(\mathrm{t})}\right.$ twist, $\mathbb{Z}_{2}^{(\mathrm{p})}$ permutation) eigenmodes, $\quad N_{(m, n)}(M)=N_{m}(M)\left(N_{m}(M)+(-1)^{n}\right) / 2$, which have the modular symmetry in Tables III and IV. Under the modular transformation, the wave functions in Eq. (88) transform similarly to Eq. (80) by replacing Eqs. (82) and (83) with

$$
\begin{aligned}
& \rho_{(\mathrm{t}) m(\mathrm{p}) n}(\gamma)_{\left(j^{(1)} j^{(2)}\right)\left(k^{(1)} k^{(2)}\right)} \\
& =2 \mathcal{N}_{(\mathrm{t}, \mathrm{p})}^{j^{(1)} j^{(2)}} \mathcal{N}_{(\mathrm{t}, \mathrm{p})}^{k^{(1)} k^{(2)}}\left(\rho_{(\mathrm{t}) m}(\gamma)_{\left(j^{(1)} j^{(2)}\right)\left(k^{(1)} k^{(2)}\right)}\right. \\
& \left.\quad+(-1)^{n} \rho_{(\mathrm{t}) m}(S)_{\left(j^{(1)} j^{(2)}\right)\left(k^{(1)} k^{(2)}\right)}\right)
\end{aligned}
$$

which satisfies Eq. (8) with $k=1$ and also satisfies

$$
\begin{array}{r}
\rho_{(\mathrm{t}) m(\mathrm{p}) n}(T)^{2 M}=\mathbb{I} \quad(M \in 2 \mathbb{Z}), \\
\rho_{(\mathrm{t}) m(\mathrm{p}) n}(T)^{M}=i \mathbb{I}, \quad \rho_{(\mathrm{t}) m(\mathrm{p}) n}(T)^{2 M}=-\mathbb{I}, \\
\rho_{(\mathrm{t}) m(\mathrm{p}) n}(T)^{4 M}=\mathbb{I} \quad(M \in 2 \mathbb{Z}+1),
\end{array}
$$

corresponding to Eq. (9). Thus, the wave functions on the $\left(T_{1}^{2} \times T_{2}^{2}\right) /\left(\mathbb{Z}_{2}^{(\mathrm{t})} \times \mathbb{Z}_{2}^{(\mathrm{p})}\right)$ orbifold with magnetic flux $M \in 2 \mathbb{Z}$ and the SS phases $\left(\alpha_{1}, \alpha_{2}\right)=(0,0)$ behave as modular forms of weight 1 , and then they transform as

TABLE IV. Number of ( $\mathbb{Z}_{2}^{(\mathrm{t})}$ twist, $\mathbb{Z}_{2}^{(\mathrm{p})}$ permutation) eigenmodes, $N_{(m, n)}(M)$, on the $\left(T_{1}^{2} \times T_{2}^{2}\right) /\left(\mathbb{Z}_{2}^{(\mathrm{t})} \times \mathbb{Z}_{2}^{(\mathrm{p})}\right)$ orbifold with $M=$ odd and $\left(\alpha_{1}, \alpha_{2}\right)=(1 / 2,1 / 2)$, and the order of $T$. The three generations are boxed.

\begin{tabular}{lccccc}
\hline \hline & $M$ & 1 & 3 & 5 & 7 \\
\hline (even, even): $N_{(0,0)}(M)$ & $(M-1)(M+1) / 8$ & 0 & 1 & 3 & 6 \\
(even, odd): $N_{(0,1)}(M)$ & $(M-1)(M-3) / 8$ & 0 & 0 & 1 & 3 \\
(odd, even): $N_{(1,0)}(M)$ & $(M+1)(M+3) / 8$ & 1 & 3 & 6 & 10 \\
(odd, odd): $N_{(1,1)}(M)$ & $(M+1)(M-1) / 8$ & 0 & 1 & 3 & 6 \\
order $h$ of $T\left(T^{h}=\mathbb{I}\right)$ & $4 M$ & 4 & 12 & 20 & 28 \\
\hline \hline
\end{tabular}

$N_{(m, n)}(M)$-dimensional representations, as shown in Table III. Similarly, the wave functions with magnetic flux $M \in 2 \mathbb{Z}+1$ and the SS phases $\left(\alpha_{1}, \alpha_{2}\right)=(1 / 2,1 / 2)$ also behave as modular forms of weight 1 , and then they transform as $N_{(m, n)}(M)$-dimensional representations, as shown in Table IV.

In the next section, we show the specific modular flavor groups of the three-generation modes on the magnetized orbifolds of $T^{2} \times T^{2}$.

\section{MODULAR FLAVOR GROUPS OF THREE-GENERATION MODES ON MAGNETIZED ORBIFOLDS OF $T^{2} \times T^{2}$}
A. $\left(T_{1}^{2} \times T_{2}^{2}\right) /\left(\mathbb{Z}_{2}^{(\mathbf{t})} \times \mathbb{Z}_{2}^{(\mathbf{p})}\right)$ orbifold

First, we consider the three-generation modes on the magnetized $\left(T_{1}^{2} \times T_{2}^{2}\right) /\left(\mathbb{Z}_{2}^{(\mathrm{t})} \times \mathbb{Z}_{2}^{(\mathrm{p})}\right)$ orbifold in Tables III and IV.

As shown in Table III, we can obtain four models with three-generation modes on the $\left(T_{1}^{2} \times T_{2}^{2}\right) /\left(\mathbb{Z}_{2}^{(\mathrm{t})} \times \mathbb{Z}_{2}^{(\mathrm{p})}\right)$ orbifold with $M=$ even and $\left(\alpha_{1}, \alpha_{2}\right)=(0,0):(M, m, n)=$ $(2,0,0),(4,0,1),(6,1,0)$, and $(8,1,1)$. They can be representations of $\Delta^{\prime}\left(6 M^{2}\right)$, which are the double covering groups of $\Delta\left(6 M^{2}\right)$, similar to that shown in Sec. IV A. Namely, if Eq. (64) is also satisfied ${ }^{13}$ in addition to Eqs. (8) and (9) with $k=1$ and $N=2 M$, the generators

$$
\begin{aligned}
& a=S T^{2} S T^{4}, \quad a^{\prime}=S T^{2} S^{-1} T^{-2}, \\
& b=T^{\frac{M}{2}+3} S^{\frac{3}{2} M-1} T^{M}, \quad c=S T^{M-2} S T^{\frac{3}{2} M-1}(M=4 s),
\end{aligned}
$$

$a=S T^{2} S T^{4}, \quad a^{\prime}=S T^{2} S^{-1} T^{-2}$,

$b=T^{\frac{M}{2}} S^{\frac{3}{2} M} T^{M}, \quad c=S T^{M} S T^{\frac{3}{2} M}(M=2(2 s-1))$,

where $s \in \mathbb{Z}$, satisfy

$$
\begin{aligned}
a^{M} & =a^{\prime M}=b^{3}=c^{4}=\mathbf{1}, \\
a a^{\prime} & =a^{\prime} a, \quad c b c^{-1}=b^{-1}, \quad b a b^{-1}=a^{-1} a^{\prime-1}, \\
b a^{\prime} b^{-1} & =a, \quad c a c^{-1}=a^{-1}, \quad c a^{\prime} c^{-1}=a^{-1},
\end{aligned}
$$

which means that the generators in Eq. (93) are those of $\Delta^{\prime}\left(6 M^{2}\right) \simeq\left(Z_{M} \times Z_{M}\right) \rtimes Z_{3} \rtimes Z_{4} \simeq \Delta\left(3 M^{2}\right) \rtimes Z_{4}$, where $a^{\left({ }^{\prime}\right)}$, $b, c$ denote those of $Z_{M}^{\left({ }^{\prime}\right)}, Z_{3}, Z_{4}$, respectively. Actually, all of the following $S$ and $T$ transformation matrices for $(M, m, n)=(2,0,0),(4,0,1),(6,1,0)$, and $(8,1,1)$ satisfy Eq. (64) since they can be written as Eq. (70).

\footnotetext{
${ }^{13}$ When $M=1,2$, Eq. (64) is automatically satisfied by considering Eq. (8) (see Appendix C for details).
} 
The $S$ and $T$ transformation matrices for $(M, m, n)=$ $(2,0,0)$ are given by

$$
S=\frac{i}{2}\left(\begin{array}{ccc}
1 & \sqrt{2} & 1 \\
\sqrt{2} & 0 & -\sqrt{2} \\
1 & -\sqrt{2} & 1
\end{array}\right), \quad T=\left(\begin{array}{ccc}
1 & & \\
& i & \\
& & -1
\end{array}\right) .
$$

The $S$ and $T$ transformation matrices for $(M, m, n)=$ $(4,0,1)$ are given by

$S=-\frac{i}{2}\left(\begin{array}{ccc}1 & \sqrt{2} & 1 \\ \sqrt{2} & 0 & -\sqrt{2} \\ 1 & -\sqrt{2} & 1\end{array}\right), \quad T=e^{\pi i / 4}\left(\begin{array}{lll}1 & & \\ & e^{3 \pi i / 4} & \\ & & -1\end{array}\right)$.

The $S$ and $T$ transformation matrices for $(M, m, n)=$ $(6,1,0)$ are given by

$$
S=-\frac{i}{2}\left(\begin{array}{ccc}
1 & \sqrt{2} & 1 \\
\sqrt{2} & 0 & -\sqrt{2} \\
1 & -\sqrt{2} & 1
\end{array}\right), \quad T=e^{\pi i / 3}\left(\begin{array}{ccc}
1 & \\
& i & \\
& & -1
\end{array}\right) .
$$

The $S$ and $T$ transformation matrices for $(M, m, n)=$ $(6,1,0)$ are given by

$$
S=\frac{i}{2}\left(\begin{array}{ccc}
1 & \sqrt{2} & 1 \\
\sqrt{2} & 0 & -\sqrt{2} \\
1 & -\sqrt{2} & 1
\end{array}\right), \quad T=e^{5 \pi i / 8}\left(\begin{array}{ccc}
1 & & \\
& e^{5 \pi i / 8} & \\
& & -1
\end{array}\right) .
$$

Note that since the $T$ matrix in Eq. (98) also satisfies $T^{4}=e^{4 \pi i / 3} \mathbb{I}$, this can be the $Z_{3}$ generator, $d=T^{4}$, which commutes with all of the generators in Eq. (94), and also the generators $a$ and $a^{\prime}$ in Eq. (94) satisfy $a^{2}=a^{\prime 2}=\mathbf{1}$. Thus, the three-generation modes for $(M, m, n)=(2,0,0)$, $(4,0,1),(6,1,0)$, and $(8,1,1)$ are transformed under the modular transformation as the three-dimensional representations of $S_{4}^{\prime} \simeq \Delta^{\prime}(24), \Delta^{\prime}(96), S_{4}^{\prime} \times Z_{3}$, and $\Delta^{\prime}(384)$, respectively.

We also comment on the anomaly of these modular flavor groups. From Eqs. (8) with $k=1$, (9) with $N=2 M$, (93), (94), and (95), similarly, we can obtain

$$
\begin{aligned}
& \operatorname{det}(a)=\operatorname{det}\left(a^{\prime}\right)=\operatorname{det}(b)=1, \\
& \operatorname{det}(c)=\left\{\begin{array}{ll}
\operatorname{det}(T)^{\frac{M}{2}+3} & (M=4 s), \\
\operatorname{det}(T)^{\frac{M}{2}+6} & (M=2(2 s-1)),
\end{array}, \operatorname{det}(c)^{4}=1 .\right.
\end{aligned}
$$

All of Eqs. (96)-(99) satisfy Eq. (100) and $\operatorname{det}(c)=i$. In Eq. (98), $\operatorname{det}(d)=\operatorname{det}(T)^{4}=1$ is also satisfied. Thus, in all cases, only $Z_{4}$ symmetry (generated by $c$ ) can be anomalous, and then $A_{4} \simeq \Delta(12), \Delta(48), A_{4} \times Z_{3}$, and $\Delta(192)$ remain anomaly free.

As shown in Table IV, we can obtain four models with three-generation modes on the $\left(T_{1}^{2} \times T_{2}^{2}\right) /\left(\mathbb{Z}_{2}^{(\mathrm{t})} \times \mathbb{Z}_{2}^{(\mathrm{p})}\right)$ orbifold with $M=$ odd and $\left(\alpha_{1}, \alpha_{2}\right)=(1 / 2,1 / 2)$ : $(M, m, n)=(3,1,0),(5,0,0),(5,1,1)$, and $(7,0,1)$. We note that all of the following $S$ and $T$ transformation matrices satisfy Eqs. (8) and (92) with $k=1$. First, from the $S$ and $T$ transformation matrices for $(M, m, n)=(3,1,0)$,

$$
\begin{aligned}
& S=-\frac{i}{3}\left(\begin{array}{ccc}
1 & 2 e^{\pi i / 3} & 2 e^{2 \pi i / 3} \\
2 e^{-\pi i / 3} & 1 & -2 e^{\pi i / 3} \\
2 e^{-2 \pi i / 3} & -2 e^{-\pi i / 3} & 1
\end{array}\right), \\
& T=\left(\begin{array}{ccc}
e^{\pi i / 6} & \\
& e^{5 \pi i / 6} & \\
& & e^{9 \pi i / 6}
\end{array}\right),
\end{aligned}
$$

TABLE V. Flavor groups of the three-generation modes $\left(M^{(1)}, m_{1}: M^{(2)}, m_{2}\right)$ which satisfy $N_{m_{1}}\left(M^{(1)}\right)=3$ and $N_{m_{2}}\left(M^{(2)}\right)=1$, on the magnetized $T_{1}^{2} / \mathbb{Z}_{2}^{\left(\mathrm{t}_{1}\right)} \times T^{2} / \mathbb{Z}_{2}^{\left(\mathrm{t}_{2}\right)}$ orbifold. The anomaly-free subgroups are also shown.

\begin{tabular}{lccc}
\hline \hline$\left(M^{(1)}, m_{1}: M^{(2)}, m_{2}\right)$ & orders $\left(h_{S}, h_{T}\right)$ of $S$ and $T\left(S^{h_{S}}=T^{h_{T}}=\mathbb{I}\right)$ & modular flavor group & anomaly-free group \\
\hline$(4,0: 4,1)$ & $(2,8)$ & $\Delta(96)$ & $\Delta(96)$ \\
$(4,0: 1,1)$ & $(2,8)$ & $\Delta(96)$ & $\Delta(96)$ \\
$(4,0: 3,0)$ & $(4,24)$ & $\Delta^{\prime}(96) \times Z_{3}$ & $\Delta(48) \times Z_{3}$ \\
$(8,1: 4,1)$ & $(4,16)$ & $\Delta^{\prime}(384)$ & $\Delta(192)$ \\
$(8,1: 1,1)$ & $(4,16)$ & $\Delta^{\prime}(384)$ & $\Delta(192)$ \\
$(8,1: 3,0)$ & $(2,48)$ & $\Delta(384) \times Z_{3}$ & $\Delta(384) \times Z_{3}$ \\
$(5,1: 4,1)$ & $(4,20)$ & $A_{5} \times Z_{4}$ & $A_{5}$ \\
$(5,1: 1,1)$ & $(4,20)$ & $A_{5} \times Z_{4}$ & $A_{5}$ \\
$(5,1: 3,0)$ & $(2,15)$ & $A_{5} \times Z_{3}$ & $A_{5} \times Z_{3}$ \\
$(7,0: 4,1)$ & $(2,7)$ & $P S L\left(2, Z_{7}\right)$ & $P S L\left(2, Z_{7}\right)$ \\
$(7,0: 1,1)$ & $(2,7)$ & $P S L\left(2, Z_{7}\right)$ & $P S L\left(2, Z_{7}\right)$ \\
$(7,0: 3,0)$ & $(4,84)$ & $P S L\left(2, Z_{7}\right) \times Z_{3} \times Z_{4}$ & $P S L\left(2, Z_{7}\right) \times Z_{3}$ \\
\hline \hline
\end{tabular}


we can obtain the generators

$$
a=S T^{9}, \quad b=S T, \quad c=T^{3},
$$

which satisfy

$a^{2}=b^{3}=(a b)^{3}=c^{4}=\mathbf{1}, \quad a c=c a, \quad b c=c b$, which means that the generators in Eq. (102) are those of $A_{4} \times Z_{4}$. Thus, the three-generation modes $(M, m, n)=$ $(3,1,0)$ are transformed under the modular transformation as the three-dimensional representations of $A_{4} \times Z_{4}$.

Second, from the $S$ and $T$ transformation matrices for $(M, m, n)=(5,0,0)$,

$$
\begin{aligned}
& S=\frac{4 i}{5}\left(\begin{array}{ccc}
A^{2} & \sqrt{2} e^{\pi i / 5} A B & e^{2 \pi i / 5} B^{2} \\
\sqrt{2} e^{-\pi i / 5} A B & B^{2}-A^{2} & -\sqrt{2} e^{\pi i / 5} A B \\
e^{-2 \pi i / 5} B^{2} & -\sqrt{2} e^{-\pi i / 5} A B & A^{2}
\end{array}\right), \quad T=\left(\begin{array}{ccc}
e^{\pi i / 10} & \\
& e^{5 \pi i / 10} & \\
& e^{9 \pi i / 10}
\end{array}\right) \\
& A=\cos \left(\frac{\pi}{10}\right), \quad B=\cos \left(\frac{3 \pi}{10}\right),
\end{aligned}
$$

we can obtain the generators

$$
a=S T^{5}, \quad b=S T, \quad c=T^{5},
$$

which satisfy

$$
a^{2}=b^{3}=(a b)^{5}=c^{4}=\mathbf{1}, \quad a c=c a, \quad b c=c b,
$$

which means that the generators in Eq. (105) are those of $A_{5} \times Z_{4}$. Thus, the three-generation modes $(M, m, n)=$ $(5,0,0)$ are transformed under the modular transformation as the three-dimensional representations of $A_{5} \times Z_{4}$.

Third, similarly, from the $S$ and $T$ transformation matrices for $(M, m, n)=(5,1,1)$,

$$
\begin{aligned}
& S=-\frac{2 i}{5}\left(\begin{array}{ccc}
2\left(A^{2}-B^{2}\right) & -\sqrt{2} e^{\pi i / 5}(A+B) & -\sqrt{2} e^{2 \pi i / 5}(A+B) \\
-\sqrt{2} e^{-\pi i / 5}(A+B) & A-1 & e^{\pi i / 5}(B+1) \\
-\sqrt{2} e^{-2 \pi i / 5}(A+B) & e^{-\pi i / 5}(B+1) & A-1
\end{array}\right), \\
& A=\sin \left(\frac{\pi}{10}\right), \quad B=\sin \left(\frac{3 \pi}{10}\right), \\
& T=\left(\begin{array}{ccc}
e^{5 \pi i / 10} & & \\
& e^{13 \pi i / 10} & \\
& & e^{17 \pi i / 10}
\end{array}\right),
\end{aligned}
$$

we can obtain the generators in Eq. (105) satisfying Eq. (106). Thus, the three-generation modes $(M, m, n)=(5,1,1)$ are also transformed under the modular transformation as the three-dimensional representations of $A_{5} \times Z_{4}$.

Fourth, from the $S$ and $T$ transformation matrices for $(M, m, n)=(7,0,1)$,

$$
\begin{aligned}
& S=\frac{4 i}{7}\left(\begin{array}{ccc}
A D-B^{2} & -e^{\frac{\pi i}{7}}\left(A^{2}+B C\right) & -e^{\frac{2 \pi i}{7}}(A B+C D) \\
-e^{-\frac{\pi i}{7}}\left(A^{2}+B C\right) & A B-C^{2} & e^{\frac{\pi i}{7}}\left(B^{2}+A C\right) \\
-e^{-\frac{2 \pi i}{7}}(A B+C D) & e^{-\frac{\pi i}{7}}\left(B^{2}+A C\right) & B D-A^{2}
\end{array}\right), \\
& A=\cos \left(\frac{\pi}{14}\right), \quad B=\cos \left(\frac{3 \pi}{14}\right), \quad C=\cos \left(\frac{5 \pi}{14}\right), \quad D=\cos \left(\frac{9 \pi}{14}\right) \text {, } \\
& T=\left(\begin{array}{ccc}
e^{5 \pi i / 14} & & \\
& e^{13 \pi i / 14} & \\
& & e^{17 \pi i / 14}
\end{array}\right),
\end{aligned}
$$


which also satisfy Eq. (76), we can obtain the generators

$$
a=S T^{21}, \quad b=S^{3} T^{3}, \quad c=T^{7},
$$

which satisfy

$a^{2}=b^{4}=(a b)^{7}=\left(a^{-1} b^{-1} a b\right)^{4}=c^{4}=\mathbf{1}$,

$a c=c a, \quad b c=c b$,

which means that the generators in Eq. (109) are those of $\operatorname{PSL}\left(2, Z_{7}\right) \times Z_{4}$. Thus, the three-generation modes $(M, m, n)=(7,0,1)$ are transformed under the modular transformation as the three-dimensional representations of $\operatorname{PSL}\left(2, Z_{7}\right) \times Z_{4}$.

Finally, we also comment on the anomaly of these modular flavor groups. From Eqs. (101)-(109), and also Eqs. (8) and (92) with $k=1$, we can obtain

$\operatorname{det}(a)=\operatorname{det}(b)=1, \quad \operatorname{det}(c)=\operatorname{det}(i \mathbb{I})=-i, \quad \operatorname{det}(c)^{4}=1$.

Thus, in all of the above cases, only $Z_{4}$ symmetry (generated by $c$ ) can be anomalous, and then $A_{4}, A_{5}$ and $\operatorname{PSL}\left(2, Z_{7}\right)$ remain anomaly free.

Therefore, on the magnetized $\left(T_{1}^{2} \times T_{2}^{2}\right) /\left(\mathbb{Z}_{2}^{(\mathrm{t})} \times \mathbb{Z}_{2}^{(\mathrm{p})}\right)$ orbifold, we can obtain three-dimensional representations of all of the double covering groups of $\Gamma_{4} \simeq S_{4}$, $\Gamma_{8} \supset \Delta(96)$, and $\Gamma_{16} \supset \Delta(384)$ for even magnetic fluxes and $Z_{4}$ central extended groups of $\Gamma_{3} \simeq \operatorname{PSL}\left(2, \mathbb{Z}_{3}\right) \simeq A_{4}$, $\Gamma_{5} \simeq \operatorname{PSL}\left(2, \mathbb{Z}_{5}\right) \simeq A_{5}, \Gamma_{7} \simeq \operatorname{PSL}\left(2, \mathbb{Z}_{7}\right)$ for odd magnetic fluxes.

\section{B. Other $T_{1}^{2} / \mathbb{Z}_{2}^{\left(\mathrm{t}_{1}\right)} \times T^{2} / \mathbb{Z}_{2}^{\left(\mathrm{t}_{2}\right)}$ orbifolds}

Finally, we consider the three-generation modes on the magnetized $T_{1}^{2} / \mathbb{Z}_{2}^{\left(\mathrm{t}_{1}\right)} \times T^{2} / \mathbb{Z}_{2}^{\left(\mathrm{t}_{2}\right)}$ orbifold, where $T_{1}^{2} / \mathbb{Z}_{2}^{\left(\mathrm{t}_{1}\right)}$ and $T^{2} / \mathbb{Z}_{2}^{\left(\mathrm{t}_{2}\right)}$ are not identified. In order to obtain $N_{m_{1}}\left(M^{(1)}\right) N_{m_{2}}\left(M^{(2)}\right)=3$ on the magnetized $T_{1}^{2} / \mathbb{Z}_{2}^{\left(\mathrm{t}_{1}\right)} \times$ $T^{2} / \mathbb{Z}_{2}^{\left(\mathrm{t}_{2}\right)}$ orbifold, we can only consider $N_{m_{1}}\left(M^{(1)}\right)=3$ and $N_{m_{2}}\left(M^{(2)}\right)=1$. Then, from Tables I and II we can consider 12 patterns, listed in Table V. The corresponding finite modular subgroups, which can be found by considering $Z=-(-1)^{m_{1}+m_{2}} \mathbf{1}$ and Eqs. (84)-(87), ${ }^{14}$ are also listed in Table V. The $S$ and $T$ transformation matrices for the $\mathbb{Z}_{2}^{(\mathrm{t})}$ odd modes with $M=1$ as well as the $\mathbb{Z}_{2}^{(\mathrm{t})}$-odd modes with $M=4$ are given by

$$
S=e^{3 \pi i / 4}, \quad T=e^{\pi i / 4},
$$

\footnotetext{
${ }^{14}$ There is an exception in Eq. (85); Eq. (85) for the $M^{(1)}=4$ singlet mode-that is, the $\mathbb{Z}_{2}^{\left(\mathrm{t}_{1}\right)}$-odd mode of $M^{(1)}=4-$ corresponds to Eq. (87) with $M^{(1)}=1$.
}

and those for the $\mathbb{Z}_{2}^{(\mathrm{t})}$-even modes with $M=3$ are given by

$$
S=e^{\pi i / 4}, \quad T=e^{\pi i / 12},
$$

while those for $N_{m_{1}}\left(M^{(1)}\right)=3$ modes were given in Sec. IV. Then, we can find the specific modular flavor groups as shown in Table V. We also show their anomalyfree subgroups in Table $\mathrm{V}$.

\section{CONCLUSION}

We have studied the modular symmetry of wave functions on magnetized orbifolds: the $T^{2} / \mathbb{Z}_{2}^{(\mathrm{t})}$ twisted orbifold, $T_{1}^{2} / \mathbb{Z}_{2}^{\left(\mathrm{t}_{1}\right)} \times T^{2} / \mathbb{Z}_{2}^{\left(\mathrm{t}_{2}\right)}$ twisted orbifold, and $\mathbb{Z}_{2}^{(\mathrm{p})}$ permutation orbifold, i.e., $\left(T_{1}^{2} \times T_{2}^{2}\right) /\left(\mathbb{Z}_{2}^{(\mathrm{t})} \times \mathbb{Z}_{2}^{(\mathrm{p})}\right)$ orbifold, with the Scherk-Schwarz phases. We found that we can consider the modular symmetry of not only wave functions with magnetic flux $M=$ even and vanishing SS phases $\left(\alpha_{1}, \alpha_{2}\right)=(0,0)$, but also those with magnetic flux $M=$ odd and SS phases $\left(\alpha_{1}, \alpha_{2}\right)=(1 / 2,1 / 2)$.

Moreover, we investigated the specific modular flavor groups for three-generation modes on the magnetized orbifolds. The three-generation modes on the magnetized $T^{2} / \mathbb{Z}_{2}^{(\mathrm{t})}$ twisted orbifold with magnetic flux $M=4,8$ are three-dimensional representations of $\tilde{\Delta}(96), \tilde{\Delta}(384)$, which are quadruple covering groups of $\Delta(96), \Delta(384)$, respectively. Among them, only $Z_{8}$ symmetries can be anomalous, and then $\Delta(48), \Delta(192)$ are anomaly free, respectively. Note that since the anomalous $Z_{8}$ symmetry is a discrete subgroup of $U(1)$, it can be canceled by the Green-Schwarz mechanism. The three-generation modes on the magnetized $T^{2} / \mathbb{Z}_{2}^{(\mathrm{t})}$ twisted orbifold with magnetic flux $M=5,7$ are three-dimensional representations of $A_{5} \times Z_{8}, P S L\left(2, Z_{7}\right) \times Z_{8}$, respectively. Among them, only $Z_{8}$ symmetries can be anomalous, and then $A_{5}$ and $\operatorname{PSL}\left(2, Z_{7}\right)$ are anomaly free, respectively. Similarly, the three-generation modes on the magnetized $\left(T_{1}^{2} \times T_{2}^{2}\right) /$ $\left(\mathbb{Z}_{2}^{(\mathrm{t})} \times \mathbb{Z}_{2}^{(\mathrm{p})}\right)$ orbifold are the corresponding three-dimensional representations of the double covering groups of $\Gamma_{N}$ for $N=4,8,16$ and $Z_{4}$ central extended groups of $\Gamma_{N}$ for $N=3,5,7$, provided in Ref. [29]. Among them, only $Z_{8}$ symmetries can be anomalous, and then $\Delta\left(3 M^{2}\right)$ for $N=2 M=4,8,16, A_{4}$ for $N=3, A_{5}$ for $N=5$, and $\operatorname{PSL}\left(2, Z_{7}\right)$ for $N=7$ are anomaly free. We have also shown the specific modular flavor groups of the threegeneration modes on the other distinguishable magnetized $T_{1}^{2} / \mathbb{Z}_{2}^{\left(\mathrm{t}_{1}\right)} \times T^{2} / \mathbb{Z}_{2}^{\left(\mathrm{t}_{2}\right)}$ orbifolds in Table V.

Our results on flavor symmetries of three generations are useful to understand quark and lepton masses and their mixing angles. Also, anomaly behaviors are useful (see, e.g., Ref. [45]). We will investigate realistic model building 
considering the obtained modular flavor groups in magnetized orbifold models elsewhere.

\section{ACKNOWLEDGMENTS}

T. K. was supported in part by MEXT KAKENHI Grant No. JP19H04605. H. U. was supported by Grant-in-Aid for JSPS Research Fellows No. 20J20388.

\section{APPENDIX A: SCHERK-SCHWARZ PHASES AND WILSON LINES}

Here we show that Scherk-Schwarz phases can be converted into Wilson lines through a gauge transformation 26]], and also that their modular transformations are consistent with each other.

First, let us consider the following gauge transformation:

$$
\begin{aligned}
\tilde{\psi}^{\alpha_{1}, \alpha_{2}}(z, \tau) & =e^{-i \operatorname{Re} \bar{\beta} z} \psi^{\alpha_{1}, \alpha_{2}}(z, \tau), \\
\tilde{A}(z)=A(z)-d[\operatorname{Re} \bar{\beta} z] & =\frac{\pi M}{\operatorname{Im} \tau} \operatorname{Im}\left(\left(\bar{z}-\frac{i \operatorname{Im} \tau}{\pi M} \bar{\beta}\right) d z\right),
\end{aligned}
$$

where $\beta$ is a complex number, $\psi^{\alpha_{1}, \alpha_{2}}$ satisfies Eqs. (42) and (43), and $A(z)$ is as in Eq. (11). We can regard $\frac{i \operatorname{Im} \tau}{\pi M} \beta \equiv \tilde{a}_{w}$ as the WL. Accordingly, $\chi_{1}(z)$ and $\chi_{2}(z)$, defined in Eqs. (12) and (13), are deformed as

$$
\begin{gathered}
\tilde{\chi}_{1}(z)=\frac{\pi M}{\operatorname{Im} \tau} \operatorname{Im}\left(z+\frac{i \operatorname{Im} \tau}{\pi M} \beta\right)=\chi_{1}(z)+\operatorname{Re} \beta \\
\tilde{\chi}_{2}(z)=\frac{\pi M}{\operatorname{Im} \tau} \operatorname{Im} \bar{\tau}\left(z+\frac{i \operatorname{Im} \tau}{\pi M} \beta\right)=\chi_{2}(z)+\operatorname{Re} \bar{\tau} \beta
\end{gathered}
$$

Therefore, the boundary conditions of the gaugetransformed wave function $\tilde{\psi}^{\alpha_{1}, \alpha_{2}}$ are modified from Eqs. (42) and (43) as

$$
\begin{aligned}
& \tilde{\psi}^{\alpha_{1}, \alpha_{2}}(z+1, \tau)=e^{2 \pi i \alpha_{1}-2 i \operatorname{Re} \beta} e^{i \tilde{\chi}_{1}(z)} \tilde{\psi}^{\alpha_{1}, \alpha_{2}}(z, \tau), \\
& \tilde{\psi}^{\alpha_{1}, \alpha_{2}}(z+\tau, \tau)=e^{2 \pi i \alpha_{2}-2 i \operatorname{Re} \bar{\tau} \beta} e^{i \tilde{\chi}_{2}(z)} \tilde{\psi}^{\alpha_{1}, \alpha_{2}}(z, \tau) .
\end{aligned}
$$

When we chose $\beta=-i \pi \frac{\alpha_{1} \tau-\alpha_{2}}{\operatorname{Im} \tau}$, the gauge-transformed wave function,

$$
\tilde{\psi}^{\alpha_{1}, \alpha_{2}}(z, \tau)=e^{\pi i \frac{\operatorname{lm}\left(\alpha_{1} \bar{z}-\alpha_{2}\right) z}{\operatorname{lm} \tau}} \psi^{\alpha_{1}, \alpha_{2}}(z, \tau),
$$

has the WL $M \tilde{a}_{w}=\alpha_{1} \tau-\alpha_{2}$ and vanishing SS phases $\left(\tilde{\alpha}_{1}, \tilde{\alpha}_{2}\right)=(0,0)$. That is, the SS phases $\left(\alpha_{1}, \alpha_{2}\right)$ can be converted into the WL $M \tilde{a}_{w}=\alpha_{1} \tau-\alpha_{2}$ through the gauge transformation in Eq. (A7). Actually, the $j$ th wave function can be expressed as

$$
\tilde{\psi}_{T^{2}}^{\left(j+\alpha_{1}, \alpha_{2}\right), M}(z, \tau)=e^{-\pi i \frac{\alpha_{1} \alpha_{2}}{M}} \psi_{T^{2}}^{(j+0,0), M}\left(z+\tilde{a}_{w}, \tau\right) .
$$

Next, let us consider the modular transformation. When $M=$ even $(x=0)$, the WL transforms as

$$
T\left(M \tilde{a}_{w}\right)=\alpha_{1}(\tau+1)-\left(\alpha_{1}+\alpha_{2}\right)=M \tilde{a}_{w}, \quad T=\left(\begin{array}{ll}
1 & 1 \\
0 & 1
\end{array}\right),
$$

$$
S\left(M \tilde{a}_{w}\right)=-\alpha_{2}\left(-\frac{1}{\tau}\right)-\alpha_{1}=\frac{M \tilde{a}_{w}}{-\tau}, \quad S=\left(\begin{array}{cc}
0 & 1 \\
-1 & 0
\end{array}\right),
$$

that is, it transforms as

$$
\gamma\left(M \tilde{a}_{w}\right)=\frac{M \tilde{a}_{w}}{c \tau+d}, \quad \gamma=\left(\begin{array}{ll}
a & b \\
c & d
\end{array}\right) .
$$

In this case, as mentioned in Ref. [13], the modular transformation for the wave function on the right-hand side of Eq. (A8) is the same as Eq. (33). Furthermore, in this case the gauge phase in Eq. (A7) is invariant under the modular transformation, and then the modular transformation for the gauge-transformed wave function on the lefthand side of Eq. (A7) or Eq. (A8) is the same as Eqs. (48) and (49). These are consistent. When $M=$ odd $(x=1)$, the $T$ transformation for the WL is

$T\left(M \tilde{a}_{w}\right)=\alpha_{1}(\tau+1)-\left(\alpha_{1}+\alpha_{2}-\frac{M}{2}\right)=M\left(\tilde{a}_{w}+\frac{1}{2}\right)$.

Under the $T$ transformation, the wave function with the WL on the right-hand side of Eq. (A8) is transformed as

$$
\begin{aligned}
& \psi_{T^{2}}^{(j+0,0), M}\left(z+\tilde{a}_{w}+\frac{1}{2}, \tau+1\right) \\
& =e^{\pi i j} e^{\pi i \frac{i^{2}}{M} e^{\frac{\pi i \mathrm{~m}\left(M z+\alpha_{1} \tau-\alpha_{2}\right)}{\operatorname{lm} \tau}}} \psi_{T^{2}}^{(j+0,0), M}\left(z+\tilde{a}_{w}, \tau\right) .
\end{aligned}
$$

On the other hand, in this case the gauge phase in Eq. (A7) is also transformed:

$\tilde{\psi}^{\alpha_{1}, \alpha_{2}}(z, \tau+1)=e^{\frac{\pi i}{2} M \frac{\operatorname{Im} z}{\ln \tau}} e^{\pi i \frac{\ln \left(\alpha_{1} \bar{\tau}-\alpha_{2}\right) z}{\operatorname{lm} \tau}} \psi^{\alpha_{1}, \alpha_{2}}(z, \tau+1)$.

Considering this equation and Eq. (49), the $T$ transformation for the wave function on the left-hand side of Eq. (A8) is actually consistent with Eq. (A13).

\section{APPENDIX B: $\mathbb{Z}_{N}$ SCHERK-SCHWARZ PHASES AND $\mathbb{Z}_{N}$ SHIFT MODES}

Here we show that the wave functions on the magnetized $T^{2} \simeq \mathbb{C} / \Lambda$ with the $\mathbb{Z}_{N}$ SS phases are related to the $\mathbb{Z}_{N}$-eigenmode wave functions on the magnetized full $\mathbb{Z}_{N}$ shifted orbifold of $\tilde{T}^{2} \simeq \mathbb{C} / \tilde{\Lambda}(\tilde{\Lambda}=N \Lambda)$ without the SS phases as follows. 
First, the lattice vectors $\tilde{e}_{k}(k=1,2)$ of the lattice $\tilde{\Lambda}=N \Lambda$ are written by lattice vectors of the lattice $\Lambda, e_{k}$ $(k=1,2)$, as $\tilde{e}_{k}=N e_{k}$. Then, the coordinate and modulus of $\tilde{T}^{2} \simeq \mathbb{C} / \tilde{\Lambda},(\tilde{z}, \tilde{\tau}) \equiv\left(u / \tilde{e}_{1}, \tilde{e}_{2} / \tilde{e}_{1}\right)$ are related to those of $T^{2} \simeq \mathbb{C} / \Lambda, \quad(z, \tau) \equiv\left(u / e_{1}, e_{2} / e_{1}\right) \quad$ as $\quad(\tilde{z}, \tilde{\tau})=(z / N, \tau)$, where $u$ is the coordinate of $\mathbb{C}$. Note that $\tilde{z}+1 \sim \tilde{z}$ and $\tilde{z}+\tilde{\tau} \sim \tilde{z}$ are satisfied on $\tilde{T}^{2}$.

The $\tilde{T}^{2} / \mathbb{Z}_{N}$ full shifted orbifold [13], on which the full modular symmetry remains, can be obtained by further identifying any $\mathbb{Z}_{N}$ shifted points $\tilde{z}+(r+s \tilde{\tau}) / N(\forall r$, $s \in \mathbb{Z}_{N}$ ) with $\tilde{z}$ (see also Ref. [41]). Then, the boundary conditions of the wave function on the $\tilde{T}^{2} / \mathbb{Z}_{N}$ full shifted orbifold with magnetic flux $\tilde{M}$ and vanishing SS phases are just the following two conditions:

$\psi_{\tilde{T}^{2} / \mathbb{Z}_{N}^{\left(\ell_{1}, \ell_{2}\right)}}\left(\tilde{z}+\frac{1}{N}, \tilde{\tau}\right)=e^{2 \pi i \frac{\ell_{1}}{N}} e^{\pi i \tilde{M}_{\operatorname{Im} \tilde{\tau}}^{\operatorname{Im} \tilde{\tilde{z}}}} \psi_{\tilde{T}^{2} / \mathbb{Z}_{N}^{\left(\ell_{1}, \ell_{2}\right)}}(\tilde{z}, \tilde{\tau})$,

$\psi_{\tilde{T}^{2} / \mathbb{Z}_{N}^{\left(\ell_{1}, \ell_{2}\right)}}\left(\tilde{z}+\frac{\tilde{\tau}}{N}, \tilde{\tau}\right)=e^{2 \pi i \frac{\ell_{2}}{N}} e^{\pi i \tilde{M}^{\operatorname{Im} \frac{\tilde{\tilde{n}} \tilde{\tilde{z}}}{\ln \tilde{z}}}} \psi_{\tilde{T}^{2} / \mathbb{Z}_{N}^{\left(\ell_{1}, \ell_{2}\right)}}(\tilde{z}, \tilde{\tau})$,

where $\ell_{1}, \ell_{2} \in \mathbb{Z}_{N}$ are the $\mathbb{Z}_{N}$ eigenvalues. From the above boundary conditions, $\tilde{M} / N^{2} \equiv M \in \mathbb{Z}$ should be satisfied. The above wave function on the magnetized $\tilde{T}^{2} / \mathbb{Z}_{N}$ full shifted orbifold without SS phases, $\psi_{\tilde{T}^{2} / \mathbb{Z}_{N}^{\left(\ell_{1}, \ell_{2}\right)}}^{j, M}$, can be expanded by the wave function on the magnetized $\tilde{T}^{2}$ without SS phases as

$\psi_{\tilde{T}^{2} / \mathbb{Z}_{N}^{\left(\ell_{1}, \ell_{2}\right)}}^{j, M}(\tilde{z}, \tilde{\tau})=\frac{1}{\sqrt{N}} \sum_{k=0}^{N-1} e^{-2 \pi i k \frac{\ell_{2}}{N}} \psi_{\tilde{T}^{2}}^{\left(N j+\ell_{1}\right)+k N M, N^{2} M}(\tilde{z}, \tilde{\tau})$.

Furthermore, by considering the relation $(\tilde{z}, \tilde{\tau})=$ $(z / N, \tau)$, the boundary conditions in Eqs. (B1) and (B2) correspond to those in Eqs. (42) and (43) with the $\mathbb{Z}_{N}$ SS phases $\left(\alpha_{1}, \alpha_{2}\right)=\left(\ell_{1} / N, \ell_{2} / N\right)\left(\ell_{1}, \ell_{2} \in \mathbb{Z}_{N}\right)$. Actually, the above wave function with the $\mathbb{Z}_{N}$ eigenvalue $\left(\ell_{1}, \ell_{2}\right)$ on the $\tilde{T}^{2} / \mathbb{Z}_{N}$ full shifted orbifold with magnetic flux $\tilde{M}$ and vanishing SS phases is related to the wave function on $T^{2}$ with magnetic flux $M$ and the $\mathbb{Z}_{N}$ SS phases $\left(\alpha_{1}, \alpha_{2}\right)=\left(\ell_{1} / N, \ell_{2} / N\right)$ as

$$
\begin{aligned}
& \psi_{\tilde{T}^{2} / \mathbb{Z}_{N}^{\left(\ell_{1}, \ell_{2}\right)}}^{j,}\left(\frac{z}{N}, \tau\right) \\
& =\frac{1}{\sqrt{N}} \sum_{k=0}^{N-1} e^{-2 \pi i k^{\frac{\ell_{2}}{N}}} \psi_{\tilde{T}^{2}}^{\left(N j+\ell_{1}\right)+k N M, N^{2} M}\left(\frac{z}{N}, \tau\right) \\
& =e^{2 \pi i\left(j+\frac{\ell_{1}}{N}\right) \frac{\ell_{2}}{N} / M} \psi_{T^{2}}^{\left(j+\frac{\ell_{1}}{N}, \frac{\ell_{2}}{N}\right), M}(z, \tau) .
\end{aligned}
$$

The analyses of the modular transformation are also consistent.
Similarly, the wave function on the magnetized $\tilde{T}^{2} / \mathbb{Z}_{2}$ twisted and full shifted orbifold without SS phases is related to that on the magnetized $T^{2}$ with the $\mathbb{Z}_{2}$ SS phases. Their behavior of the modular transformation are consistent.

\section{APPENDIX C: $\tilde{\Delta}\left(6 M^{2}\right)$ AS A SUBGROUP OF $\tilde{\Gamma}_{2 M}$}

Here we prove that the generators in Eq. (66) (in particular, for $M \in 4 \mathbb{Z}$ ) satisfy the algebraic relations of $\tilde{\Delta}\left(6 M^{2}\right)$ in Eq. (67), where the algebraic relations of $\tilde{\Gamma}_{2 M}$ in Eqs. (27)-(31) with $N=2 M$ and the additional relation in Eq. (64) are satisfied. Note that when we have $k / 2=$ integer [even] in Eqs. (27)-(31) with $k=$ integer [even] and $N=2 M$, which correspond to the algebraic relations of $\Gamma_{2 M}^{\prime}\left[\Gamma_{2 M}\right]$ in Eqs. (8) and (9) with $N=2 M$, we can find that the generators in Eq. (66) correspond to those in Eq. (93) [Eq. (65)] and they satisfy the algebraic relations of $\Delta^{\prime}\left(6 M^{2}\right)\left[\Delta\left(6 M^{2}\right)\right]$ in Eq. (95) [Eq. (63)].

First, by using Eqs. (27)-(30), Eq. (64) can be rewritten ${ }^{15}$ as

$$
\left(S^{7} T^{3}\right)^{3}=\left(S^{-1} T^{3}\right)^{3}=\mathbf{1}
$$

By using Eqs. (27)-(30) and (C1), the generator $a^{\prime}$ in Eq. (66) can be rewritten as

$$
\begin{aligned}
a^{\prime} & =S T^{2} S^{-1} T^{-2} \\
& =S T T S^{-1} T^{2} T^{-4} \\
& =S T T^{-2} S T^{-1} T^{-2} S T^{-1} T^{-4} \\
& =S^{-1} T^{-1} S^{-1} T^{-1} S^{4} T^{-2} S T^{-5} \\
& =T S T^{-2} S T^{-5} \\
& =T S^{-1} T^{-1} S^{4} T^{-1} S^{-1} T^{-5} \\
& =T^{2} S T S S^{4} S T S T^{-4} \\
& =T^{2} S T^{2} S^{-1} T^{-4} \\
& =T^{2}\left(S T^{2} S^{-1} T^{-2}\right) T^{-2} \\
\Leftrightarrow a^{\prime} & =T^{-2}\left(S T^{2} S T^{-2}\right) T^{2} \\
& =T^{-2} S T^{2} S^{-1} .
\end{aligned}
$$

Then, we can obtain

$$
S T^{2 p} S^{-1} T^{2 q}=\left(S T^{2} S^{-1}\right)^{p} T^{2 q}=T^{2 q} S T^{2 p} S^{-1}, \quad p, q \in \mathbb{Z},
$$

in general. Similarly, by using this relation, the generator $a$ in Eq. (66) can be rewritten as

\footnotetext{
${ }^{15}$ When we consider Eqs. (8) and (9) with $N=2 M, M=1,2$, we can check that Eq. (C1) is already satisfied.
} 


$$
\begin{aligned}
a & =S T^{2} S^{5} T^{4} \\
& =T^{4} S T^{2} S^{5} .
\end{aligned}
$$

Thus, we can obtain

$$
\begin{gathered}
a^{M}=S^{-2 M} T^{4 M} S T^{2 M} S^{-1}=1 \\
a^{\prime M}=T^{-2 M} S T^{2 M} S^{-1}=1 \\
a a^{\prime}=S T^{4} S^{5} T^{2}=a^{\prime} a
\end{gathered}
$$

by also using Eq. (31) with $N=2 M$ and $M \in 4 \mathbb{Z} .^{16}$ Furthermore, from Eq. (28) we also have

$$
\left(S^{5} T\right)^{3}=\mathbf{1} .
$$

Then, we can prove that

$$
\left(S^{2 n+3} T^{2 n-1}\right)^{3}=\mathbf{1}, n \in \mathbb{N}
$$

by mathematical induction. Thus, we can obtain the other relations in Eq. (67),

$$
\begin{gathered}
b^{3}=T^{-M}\left(T^{\frac{3}{2} M+3} S^{\frac{3}{2} M-1}\right)^{3} T^{M}=1 \\
c^{2}=S T^{M-2} S T^{\frac{3}{2} M-1} S T^{M-2} S T^{\frac{3}{2} M-1} \\
=S T^{M-2} S^{-1} T^{-1} S^{-1} T^{M-2} S T^{M-1} S^{4} \\
=S T^{M-1} S T^{M-1} S T^{M-1} \\
=\left(S^{M+3} T^{M-1}\right)^{3} S^{-3 M-6} \\
\quad=S^{M+2}, \\
\quad c^{4}=S^{4}, \\
\quad c^{8}=1, \\
=S c^{-1}=T^{2} S T^{M-2} S T^{2} S^{\frac{3}{2} M-1} T^{1-\frac{M}{2}} S^{-1} T^{2-M} S^{-1} T^{-M} S^{-1} T^{-\frac{M}{2}-4} \\
=T^{2} S T^{M+3} S^{\frac{3}{2} M-1} T^{3-M} S^{5} T S T^{-\frac{M}{2}-3} \\
=T^{2} S T^{M+3} S^{M-1} T^{M+3} S^{5} T S^{-\frac{3}{2} M+1} T^{-\frac{M}{2}-3} \\
=T^{2} S^{M-1} T^{M+3} S^{M-1} T^{M+3} S^{-M-1} T S^{-\frac{3}{2} M+1} T^{-\frac{M}{2}-3} \\
=T^{2} T^{-M-3} S^{-M+1} S^{-M-1} T S^{-\frac{3}{2} M+1} T^{-\frac{M}{2}-3} \\
=T^{-M} S^{-\frac{3}{2} M+1} T^{-\frac{M}{2}-3} \\
=b^{-1},
\end{gathered}
$$

\footnotetext{
${ }^{16}$ This is because $S^{-2 M}=1$ is satisfied only if $M \in 4 \mathbb{Z}$. However, when we consider the case that Eqs. (8) and (9) with $N=2 M$ are satisfied instead of Eqs. (27)-(31) with $N=2 M$, $S^{-2 M}=1$ is satisfied even if $M=2(2 s-1)(s \in \mathbb{Z})$.
}

$$
\begin{aligned}
b a b^{-1} & =T^{\frac{M}{2}+3} S^{\frac{3}{2} M-1} T^{M} S T^{2} S^{5} T^{4-M} S^{-\frac{3}{2} M+1} T^{-\frac{M}{2}-3} \\
& =T^{\frac{M}{2}+3} S^{\frac{3}{2} M-1} T^{4} S^{-\frac{3}{2} M-1} T^{-\frac{M}{2}-1} \\
& =T S^{-1} T^{4} S^{-1} T \\
& =T^{-2} S^{-1} T^{-3} S T S T \\
& =T^{-2} S^{-5} T^{-4} S^{-1} \\
& =a^{-1} a^{-1}
\end{aligned}
$$

$$
\begin{aligned}
b a^{\prime} b^{-1} & =T^{\frac{M}{2}+3} S^{\frac{3}{2} M-1} T^{M} S T^{2} S^{-1} T^{-2-M} S^{-\frac{3}{2} M+1} T^{-\frac{M}{2}-3} \\
& =T^{\frac{M}{2}+3} S^{\frac{3}{2} M-1} T^{-2} S^{-\frac{3}{2} M+1} T^{-\frac{M}{2}-1} \\
& =T^{-1} S^{-1} T^{-2} S^{-1} T^{-1} S^{2} T^{4} \\
& =S T S S^{2} S T S T^{4} \\
& =S T^{2} S^{5} T^{4} \\
& =a
\end{aligned}
$$

$$
\begin{aligned}
c a c^{-1} & =S T^{M-2} S T^{\frac{3}{2} M-1} S T^{2} S^{5} T^{5-\frac{3}{2} M} S^{-1} T^{2-M} S^{-1} \\
& =S T^{M-2} S^{-1} T^{-1} S^{-1} T^{2} S^{5} T^{5} S T^{2-M} S \\
& =S T^{M-1} S^{5} T^{3} S^{5} T^{5} S T^{2-M} S \\
& =S^{-1} T^{M-2} T S^{7} T^{3} S^{7} T^{2} T^{3} S T^{2-M} S^{-1} \\
& =S^{-1} T^{M-4} S T^{2} S T^{2-M} S^{-1} \\
& =T^{2} S T^{-2} S^{-1} \\
& =a^{\prime-1}
\end{aligned}
$$

$$
\begin{aligned}
c a^{\prime} c^{-1} & =S T^{M-2} S T^{\frac{3}{2} M-1} S T^{2} S^{-1} T^{-1-\frac{3}{2} M} S^{-1} T^{2-M} S^{-1} \\
& =S T^{M-2} S^{-1} T^{-1} S T^{2} S^{-1} T^{-1} S^{-1} T^{2-M} S \\
& =S T^{M-1} S^{-1} T^{4} S^{5} T^{3-M} S \\
& =S T S T^{4} S^{3} T S \\
& =T^{-1} S^{3} T^{3} S^{3} T S \\
& =T^{-1} S^{5} T^{2} S^{3} T^{-1} \\
& =T^{-2} S^{-1} T^{-1} S^{-1} S^{6} S^{-1} T^{-1} S^{-1} T^{-2} \\
& =T^{-2} S^{-5} T^{-2} S^{-1} T^{-2} \\
& =T^{-4} S^{-5} T^{-2} S^{-1} \\
& =a^{-1}
\end{aligned}
$$

Therefore, when the relation in Eq. (64) is also satisfied in addition to the algebraic relations of $\tilde{\Gamma}_{2 M}$ (in particular, for $M \in 4 \mathbb{Z}$ ), Eq. (66) can be the generators of $\tilde{\Delta}\left(6 M^{2}\right)$. Similarly, when the algebraic relations of $\Gamma_{2 M}^{\prime}\left[\Gamma_{2 M}\right]$ and also Eq. (64) are satisfied, we can find that Eq. (93) [Eq. (65)] as well as Eq. (94) [the generators in footnote 8] can be the generators of $\Delta^{\prime}\left(6 M^{2}\right)\left[\Delta\left(6 M^{2}\right)\right]$. 


\section{APPENDIX D: THREE-DIMENSIONAL MODULAR FORMS}

Here we express the three-dimensional modular forms obtained from the wave functions on magnetized orbifolds at $z=0$, which means that the modular forms can be obtained from $\mathbb{Z}_{2}$-even $(m=n=0)$ modes.

We can obtain two three-dimensional modular forms of weight $1 / 2$ from the modes $(M, m)=(4,0)$ and $(7,0)$ at $z=0$ on the magnetized $T^{2} / \mathbb{Z}_{2}^{(\mathrm{t})}$ twisted orbifold as follows:

$$
\begin{gathered}
\left(\begin{array}{l}
\vartheta_{1}^{4}(\tau) \\
\vartheta_{2}^{4}(\tau) \\
\vartheta_{3}^{4}(\tau)
\end{array}\right)=\left(\begin{array}{c}
\vartheta\left[\begin{array}{l}
0 \\
0
\end{array}\right](0,4 \tau) \\
\frac{1}{\sqrt{2}}\left(\vartheta\left[\begin{array}{c}
\frac{1}{4} \\
0
\end{array}\right](0,4 \tau)+\vartheta\left[\begin{array}{c}
\frac{3}{4} \\
0
\end{array}\right](0,4 \tau)\right) \\
\vartheta\left[\begin{array}{c}
\frac{2}{4} \\
0
\end{array}\right](0,4 \tau)
\end{array}\right)=\left(\begin{array}{c}
\vartheta\left[\begin{array}{l}
0 \\
0
\end{array}\right](0,4 \tau) \\
\sqrt{2} \vartheta\left[\begin{array}{c}
\frac{1}{4} \\
0
\end{array}\right](0,4 \tau) \\
\vartheta\left[\begin{array}{c}
\frac{2}{4} \\
0
\end{array}\right](0,4 \tau)
\end{array}\right)(M=4), \\
\left(\begin{array}{l}
\vartheta_{1}^{7}(\tau) \\
\vartheta_{2}^{7}(\tau) \\
\vartheta_{3}^{7}(\tau)
\end{array}\right)=\left(\begin{array}{c}
\frac{1}{\sqrt{2}}\left(\vartheta\left[\begin{array}{c}
\frac{1}{14} \\
-\frac{1}{2}
\end{array}\right](0,7 \tau)-\vartheta\left[\begin{array}{c}
\frac{13}{14} \\
-\frac{1}{2}
\end{array}\right](0,7 \tau)\right) \\
\frac{1}{\sqrt{2}}\left(\vartheta\left[\begin{array}{c}
\frac{3}{14} \\
-\frac{1}{2}
\end{array}\right](0,7 \tau)-\vartheta\left[\begin{array}{c}
\frac{11}{14} \\
-\frac{1}{2}
\end{array}\right](0,7 \tau)\right) \\
\frac{1}{\sqrt{2}}\left(\vartheta\left[\begin{array}{c}
\frac{5}{14} \\
-\frac{1}{2}
\end{array}\right](0,7 \tau)-\vartheta\left[\begin{array}{c}
\frac{9}{14} \\
-\frac{1}{2}
\end{array}\right](0,7 \tau)\right)
\end{array}\right)=\left(\begin{array}{c}
\sqrt{2} \vartheta\left[\begin{array}{c}
\frac{1}{14} \\
-\frac{1}{2}
\end{array}\right](0,7 \tau) \\
\sqrt{2} \vartheta\left[\begin{array}{c}
\frac{3}{14} \\
-\frac{1}{2}
\end{array}\right](0,7 \tau) \\
\sqrt{2} \vartheta\left[\begin{array}{c}
\frac{5}{14} \\
-\frac{1}{2}
\end{array}\right](0,7 \tau)
\end{array}\right)(M=7) .
\end{gathered}
$$

They are modular forms of weight $1 / 2$ for $\tilde{\Gamma}(8)$ and $\tilde{\Gamma}(56)$, respectively, and they also transform as the three-dimensional representations of $\tilde{\Delta}(96)$ and $\operatorname{PSL}\left(2, Z_{7}\right) \times Z_{8}$, respectively.

Similarly, we can obtain four three-dimensional modular forms of weight 1 , two of which are obtained from the modes $\left(M^{(1)}, m_{1}: M^{(2)}, m_{2}\right)=(4,0: 3,0)$ and $(7,0: 3,0)$ at $z_{1}=z_{2}=0$ on the magnetized $T_{1}^{2} / \mathbb{Z}_{2}^{\left(\mathrm{t}_{1}\right)} \times T^{2} / \mathbb{Z}_{2}^{\left(\mathrm{t}_{2}\right)}$ orbifold, and the other two of which are obtained from the modes $(M, m, n)=(2,0,0)$ and $(5,0,0)$ at $z_{1}=z_{2}=0$ on the magnetized $\left(T_{1}^{2} \times T_{2}^{2}\right) /\left(\mathbb{Z}_{2}^{(\mathrm{t})} \times \mathbb{Z}_{2}^{(\mathrm{p})}\right)$ orbifold, as follows:

$$
\begin{aligned}
& \left(\begin{array}{l}
\vartheta_{1}^{(4,3)}(\tau) \\
\vartheta_{2}^{(4,3)}(\tau) \\
\vartheta_{3}^{(4,3)}(\tau)
\end{array}\right)=\left(\begin{array}{c}
\sqrt{2} \vartheta\left[\begin{array}{c}
\frac{1}{6} \\
-\frac{1}{2}
\end{array}\right](0,3 \tau) \vartheta\left[\begin{array}{l}
0 \\
0
\end{array}\right](0,4 \tau) \\
2 \vartheta\left[\begin{array}{c}
\frac{1}{6} \\
-\frac{1}{2}
\end{array}\right](0,3 \tau) \vartheta\left[\begin{array}{c}
\frac{1}{4} \\
0
\end{array}\right](0,4 \tau) \\
\sqrt{2} \vartheta\left[\begin{array}{c}
\frac{1}{6} \\
-\frac{1}{2}
\end{array}\right](0,3 \tau) \vartheta\left[\begin{array}{l}
\frac{2}{4} \\
0
\end{array}\right](0,4 \tau)
\end{array}\right)\left(M^{(1)}=4, M^{(2)}=3\right) \\
& \left(\begin{array}{l}
\vartheta_{1}^{(7,3)}(\tau) \\
\vartheta_{2}^{(7,3)}(\tau) \\
\vartheta_{3}^{(7,3)}(\tau)
\end{array}\right)=\left(\begin{array}{c}
2 \vartheta\left[\begin{array}{c}
\frac{1}{6} \\
-\frac{1}{2}
\end{array}\right](0,3 \tau) \vartheta\left[\begin{array}{c}
\frac{1}{14} \\
-\frac{1}{2}
\end{array}\right](0,7 \tau) \\
2 \vartheta\left[\begin{array}{c}
\frac{1}{6} \\
-\frac{1}{2}
\end{array}\right](0,3 \tau) \vartheta\left[\begin{array}{c}
\frac{3}{14} \\
-\frac{1}{2}
\end{array}\right](0,7 \tau) \\
2 \vartheta\left[\begin{array}{c}
\frac{1}{6} \\
-\frac{1}{2}
\end{array}\right](0,3 \tau) \vartheta\left[\begin{array}{c}
\frac{5}{14} \\
-\frac{1}{2}
\end{array}\right](0,7 \tau)
\end{array}\right)\left(M^{(1)}=7, M^{(2)}=3\right),
\end{aligned}
$$




$$
\begin{aligned}
& \left(\begin{array}{c}
\vartheta_{1}^{(2,2)}(\tau) \\
\vartheta_{2}^{(2,2)}(\tau) \\
\vartheta_{3}^{(2,2)}(\tau)
\end{array}\right)=\left(\begin{array}{c}
\vartheta\left[\begin{array}{l}
0 \\
0
\end{array}\right](0,2 \tau) \vartheta\left[\begin{array}{l}
0 \\
0
\end{array}\right](0,2 \tau) \\
\sqrt{2} \vartheta\left[\begin{array}{l}
0 \\
0
\end{array}\right](0,2 \tau) \vartheta\left[\begin{array}{l}
\frac{1}{2} \\
0
\end{array}\right](0,2 \tau) \\
\vartheta\left[\begin{array}{c}
\frac{1}{2} \\
0
\end{array}\right](0,2 \tau) \vartheta\left[\begin{array}{c}
\frac{1}{2} \\
0
\end{array}\right](0,2 \tau)
\end{array}\right)\left(M^{(1)}=M^{(2)}=M=2\right) \\
& \left(\begin{array}{c}
\vartheta_{1}^{(5,5)}(\tau) \\
\vartheta_{2}^{(5,5)}(\tau) \\
\vartheta_{3}^{(5,5)}(\tau)
\end{array}\right)=\left(\begin{array}{c}
2 \vartheta\left[\begin{array}{c}
\frac{1}{10} \\
-\frac{1}{2}
\end{array}\right](0,5 \tau) \vartheta\left[\begin{array}{c}
\frac{1}{10} \\
-\frac{1}{2}
\end{array}\right](0,5 \tau) \\
2 \sqrt{2} \vartheta\left[\begin{array}{c}
\frac{1}{10} \\
-\frac{1}{2}
\end{array}\right](0,5 \tau) \vartheta\left[\begin{array}{c}
\frac{3}{10} \\
-\frac{1}{2}
\end{array}\right](0,5 \tau) \\
2 \vartheta\left[\begin{array}{c}
\frac{3}{10} \\
-\frac{1}{2}
\end{array}\right](0,5 \tau) \vartheta\left[\begin{array}{c}
\frac{3}{10} \\
-\frac{1}{2}
\end{array}\right](0,5 \tau)
\end{array}\right)\left(M^{(1)}=M^{(2)}=M=5\right)
\end{aligned}
$$

They are modular forms of weight 1 for $\Gamma(24), \Gamma(84), \Gamma(4)$, and $\Gamma(20)$ respectively, and they also transform as the threedimensional representations of $\Delta^{\prime}(96) \times Z_{3}, P S L\left(2, Z_{7}\right) \times Z_{3} \times Z_{4}, S_{4}^{\prime}$, and $A_{5} \times Z_{4}$, respectively.

[1] G. Altarelli and F. Feruglio, Rev. Mod. Phys. 82, 2701 (2010).

[2] H. Ishimori, T. Kobayashi, H. Ohki, Y. Shimizu, H. Okada, and M. Tanimoto, Prog. Theor. Phys. Suppl. 183, 1 (2010).

[3] H. Ishimori, T. Kobayashi, H. Ohki, H. Okada, Y. Shimizu, and M. Tanimoto, Lect. Notes Phys. 858, 1 (2012).

[4] D. Hernandez and A. Y. Smirnov, Phys. Rev. D 86, 053014 (2012).

[5] S. F. King and C. Luhn, Rep. Prog. Phys. 76, 056201 (2013).

[6] S. F. King, A. Merle, S. Morisi, Y. Shimizu, and M. Tanimoto, New J. Phys. 16, 045018 (2014).

[7] T. Kobayashi, H. P. Nilles, F. Ploger, S. Raby, and M. Ratz, Nucl. Phys. B768, 135 (2007).

[8] H. Abe, K. S. Choi, T. Kobayashi, and H. Ohki, Nucl. Phys. B820, 317 (2009).

[9] T. Kobayashi and S. Nagamoto, Phys. Rev. D 96, 096011 (2017).

[10] T. Kobayashi, S. Nagamoto, S. Takada, S. Tamba, and T. H. Tatsuishi, Phys. Rev. D 97, 116002 (2018).

[11] T. Kobayashi and S. Tamba, Phys. Rev. D 99, 046001 (2019).

[12] H. Ohki, S. Uemura, and R. Watanabe, Phys. Rev. D 102 , 085008 (2020).

[13] S. Kikuchi, T. Kobayashi, S. Takada, T. H. Tatsuishi, and H. Uchida, Phys. Rev. D 102, 105010 (2020).

[14] S. Kikuchi, T. Kobayashi, H. Otsuka, S. Takada, and H. Uchida, J. High Energy Phys. 11 (2020) 101.

[15] K. Hoshiya, S. Kikuchi, T. Kobayashi, Y. Ogawa, and H. Uchida, Prog. Theor. Exp. Phys. 2021, 033 B05 (2021).
[16] J. Lauer, J. Mas, and H. P. Nilles, Phys. Lett. B 226, 251 (1989); Nucl. Phys. B351, 353 (1991).

[17] W. Lerche, D. Lust, and N. P. Warner, Phys. Lett. B 231, 417 (1989).

[18] S. Ferrara, . D. Lust, and S. Theisen, Phys. Lett. B 233, 147 (1989).

[19] A. Baur, H. P. Nilles, A. Trautner, and P. K. S. Vaudrevange, Phys. Lett. B 795, 7 (2019).

[20] H. P. Nilles, S. Ramos-Snchez, and P. K. Vaudrevange, J. High Energy Phys. 02 (2020) 045.

[21] T. Kobayashi, S. Nagamoto, and S. Uemura, Prog. Theor. Exp. Phys. 2017, 023B02 (2017).

[22] Y. Kariyazono, T. Kobayashi, S. Takada, S. Tamba, and H. Uchida, Phys. Rev. D 100, 045014 (2019).

[23] T. Kobayashi and H. Otsuka, Phys. Rev. D 101, 106017 (2020).

[24] D. Cremades, L. E. Ibanez, and F. Marchesano, J. High Energy Phys. 05 (2004) 079.

[25] H. Abe, T. Kobayashi, and H. Ohki, J. High Energy Phys. 09 (2008) 043.

[26] T. H. Abe, Y. Fujimoto, T. Kobayashi, T. Miura, K. Nishiwaki, and M. Sakamoto, J. High Energy Phys. 01 (2014) 065.

[27] H. Abe, K. S. Choi, T. Kobayashi, and H. Ohki, Nucl. Phys. B814, 265 (2009).

[28] T. h. Abe, Y. Fujimoto, T. Kobayashi, T. Miura, K. Nishiwaki, M. Sakamoto, and Y. Tatsuta, Nucl. Phys. B894, 374 (2015).

[29] R. de Adelhart Toorop, F. Feruglio, and C. Hagedorn, Nucl. Phys. B858, 437 (2012). 
[30] X. G. Liu and G. J. Ding, J. High Energy Phys. 08 (2019) 134.

[31] F. Feruglio, arXiv:1706.08749; T. Kobayashi, K. Tanaka, and T. H. Tatsuishi, Phys. Rev. D 98, 016004 (2018); J. T. Penedo and S. T. Petcov, Nucl. Phys. B939, 292 (2019); J. C. Criado and F. Feruglio, SciPost Phys. 5, 042 (2018); T. Kobayashi, N. Omoto, Y. Shimizu, K. Takagi, M. Tanimoto, and T. H. Tatsuishi, J. High Energy Phys. 11 (2018) 196; P. P. Novichkov, J. T. Penedo, S. T. Petcov, and A. V. Titov, J. High Energy Phys. 04 (2019) 005; 04 (2019) 174; F. J. de Anda, S. F. King, and E. Perdomo, arXiv:1812.05620; H. Okada and M. Tanimoto, Phys. Lett. B 791, 54 (2019); T. Kobayashi, Y. Shimizu, K. Takagi, M. Tanimoto, T. H. Tatsuishi, and H. Uchida, Phys. Lett. B 794, 114 (2019); P. P. Novichkov, S. T. Petcov, and M. Tanimoto, Phys. Lett. B 793, 247 (2019).

[32] P. P. Novichkov, J. T. Penedo, and S. T. Petcov, Nucl. Phys. B963, 115301 (2021); X. G. Liu, C. Y. Yao, and G. J. Ding, arXiv:2006.10722; X. G. Liu, C. Y. Yao, B. Y. Qu, and G. J. Ding, arXiv:2007.13706; X. Wang, B. Yu, and S. Zhou, arXiv:2010.10159; C. Y. Yao, X. G. Liu, and G. J. Ding, arXiv:2011.03501.

[33] Y. Almumin, M. C. Chen, V. Knapp-Pérez, S. RamosSánchez, M. Ratz, and S. Shukla, J. High Energy Phys. 05 (2021) 078.
[34] Y. Tatsuta, arXiv:2104.03855.

[35] R. C. Gunning, Lectures on Modular Forms (Princeton University Press, Princeton, NJ, 1962).

[36] B. Schoeneberg, Elliptic Modular Functions (SpringerVerlag, Berlin, 1974).

[37] N. Koblitz, Introduction to Elliptic Curves and Modular Forms (Springer-Verlag, Berlin, 1984).

[38] J. H. Bruinier, G. V. D. Geer, G. Harder, and D. Zagier, The 1-2-3 of Modular Forms (Springer, New York, 2008).

[39] G. Shimura, Ann. Math. 97, 440 (1973).

[40] J. F. Duncan and D. A. Mcgady, arXiv:1806.09875.

[41] Y. Fujimoto, T. Kobayashi, T. Miura, K. Nishiwaki, and M. Sakamoto, Phys. Rev. D 87, 086001 (2013).

[42] J. A. Escobar and C. Luhn, J. Math. Phys. (N.Y.) 50, 013524 (2009).

[43] R. de Adelhart Toorop, F. Feruglio, and C. Hagedorn, Phys. Lett. B 703, 447 (2011).

[44] T. Araki, T. Kobayashi, J. Kubo, S. Ramos-Sanchez, M. Ratz, and P. K. S. Vaudrevange, Nucl. Phys. B805, 124 (2008).

[45] T. Kobayashi, Y. Shimizu, K. Takagi, M. Tanimoto, and T.H. Tatsuishi, J. High Energy Phys. 02 (2020) 097; Phys. Rev. D 100, 115045 (2019); 101, 039904(E) (2020). 